\title{
Counting Rational Points on Algebraic Varieties
}

\author{
T.D. Browning ${ }^{1}$, D.R. Heath-Brown ${ }^{2}$ and P. Salberger ${ }^{3}$ \\ ${ }^{1,2}$ Mathematical Institute, 24-29 St. Giles', Oxford OX1 3LB \\ ${ }^{3}$ Chalmers University of Technology, Göteborg SE-412 96 \\ ${ }^{1}$ browning@maths.ox.ac.uk, ${ }^{2}$ rhb@maths.ox.ac.uk, ${ }^{3}$ salberg@math.chalmers.se
}

\begin{abstract}
For any $N \geq 2$, let $Z \subset \mathbb{P}^{N}$ be a geometrically integral algebraic variety of degree $d$. This paper is concerned with the number $N_{Z}(B)$ of $\mathbb{Q}$-rational points on $Z$ which have height at most $B$. For any $\varepsilon>0$ we establish the estimate

$$
N_{Z}(B)=O_{d, \varepsilon, N}\left(B^{\operatorname{dim} Z+\varepsilon}\right),
$$

provided that $d \geq 6$. As indicated, the implied constant depends at most upon $d, \varepsilon$ and $N$.

Mathematics Subject Classification (2000): 11G35 (14G05)
\end{abstract}

\section{Introduction}

For any $n \geq 2$, let $F \in \mathbb{Z}\left[X_{0}, \ldots, X_{n}\right]$ be a form of degree $d$, that produces an integral hypersurface $F=0$ in $\mathbb{P}^{n}$. Throughout this paper we shall take integrality to mean geometric integrality, and likewise we shall say that a polynomial is irreducible if it is absolutely irreducible. Let $|\mathbf{x}|$ denote the norm $\max _{0 \leq i \leq n}\left|x_{i}\right|$ on $\mathbb{R}^{n+1}$, and define the quantity

$$
N(F ; B)=\#\left\{\mathbf{x} \in \mathbb{Z}^{n+1}: F(\mathbf{x})=0 \text {, h.c.f. }\left(x_{0}, \ldots, x_{n}\right)=1,|\mathbf{x}| \leq B\right\},
$$

for any $B \geq 1$. This paper is motivated by the following conjecture due to the second author [14, Conjecture 2].

Conjecture 1. Let $\varepsilon>0$ and suppose that $F \in \mathbb{Z}\left[X_{0}, \ldots, X_{n}\right]$ is an irreducible form of degree $d \geq 2$. Then we have

$$
N(F ; B)=O_{d, \varepsilon, n}\left(B^{n-1+\varepsilon}\right) .
$$

In all that follows the implied constant in any estimate is absolute unless explicitly indicated otherwise. In the case of Conjecture 1 , the constant is clearly permitted to depend only upon $d, \varepsilon$ and $n$.

Conjecture 1 has already been established in the cases $n \leq 3$ [14, Theorems 3 and 9] and in the case $d=2$ for any $n \geq 2$ [14, Theorem 2]. Moreover, upon 
combining recent work of Broberg and the third author [3] with work of the first two authors on cubic threefolds [4, Theorem 3], the conjecture has also been established in the case $n=4$. There exist examples for every $n \geq 3$ and $d \geq 2$ for which $N(F ; B) \gg B^{n-1}$. To see this it suffices to consider forms of the shape $X_{0} F_{0}-X_{1} F_{1}$, with $F_{0}, F_{1} \in \mathbb{Z}\left[X_{0}, \ldots, X_{n}\right]$ of degree $d-1$. This shows that Conjecture 1 is essentially best possible.

It is frequently more useful to have estimates for the corresponding problem in which $Z \subset \mathbb{P}^{N}$ is an arbitrary integral algebraic variety. Throughout our work we shall always assume that $Z$ is a proper subvariety of $\mathbb{P}^{N}$ and that the ideal of $Z$ is generated by forms defined over $\overline{\mathbb{Q}}$. In practice the most interesting situation is when the variety under consideration is the zero locus of forms which are all defined over $\mathbb{Q}$, as in the statement of Conjecture 1 . Indeed, whenever the ideal of $Z$ is not invariant under the action of the absolute galois group $\operatorname{Gal}(\overline{\mathbb{Q}} / \mathbb{Q})$, the set of rational points on $Z$ automatically lies on a proper subvariety of $Z$.

Let $\mathbf{x}=\left(x_{0}, \ldots, x_{N}\right) \in \mathbb{Z}^{N+1}$ be any vector such that h.c.f. $\left(x_{0}, \ldots, x_{N}\right)=1$. Then we let $x=[\mathbf{x}]$ denote the corresponding point in $\mathbb{P}^{N}(\mathbb{Q})$, and write $H(x)=$ $|\mathbf{x}|$ for its height. Conversely, we shall always represent a point $x \in \mathbb{P}^{N}(\mathbb{Q})$ by an $(N+1)$-tuple $\mathbf{x}=\left(x_{0}, \ldots, x_{N}\right) \in \mathbb{Z}^{N+1}$ such that h.c.f. $\left(x_{0}, \ldots, x_{N}\right)=1$. We proceed by defining the counting function

$$
N_{Z}(B)=\#\left\{x \in Z \cap \mathbb{P}^{N}(\mathbb{Q}): H(x) \leq B\right\},
$$

for any $B \geq 1$ and any algebraic variety $Z \subset \mathbb{P}^{N}$. Whenever $X \subset \mathbb{P}^{n}$ is a hypersurface defined by the equation $F=0$, this definition of $N_{X}(B)$ is consistent with the definition of $N(F ; B)$. Indeed, we then have

$$
N_{X}(B)=\frac{1}{2} N(F ; B)
$$

since $\mathbf{x}$ and $-\mathbf{x}$ represent the same point in projective space. Note that a hypersurface in $\mathbb{P}^{n}$ has dimension $n-1$. At this point it is worth highlighting a certain convention concerning the letters $n, N$ that we shall follow throughout this paper. Whereas $N$ will be used to denote the ambient dimension of a projective variety with arbitrary codimension, $n$ will always be used to denote the ambient dimension of a hypersurface. We may now phrase a further conjecture, which generalises Conjecture 1 to arbitrary algebraic varieties.

Conjecture 2. Let $\varepsilon>0$ and suppose that $Z \subset \mathbb{P}^{N}$ is an integral variety of degree $d \geq 2$ and dimension $m$. Then we have

$$
N_{Z}(B)=O_{d, \varepsilon, N}\left(B^{m+\varepsilon}\right) \text {. }
$$

Our first result enables us to relate Conjecture 1 and Conjecture 2. By employing a straightforward birational projection argument, we shall establish the following result in $\S 3$.

Theorem 1. Let $\varepsilon>0$. Suppose that there exists $\theta_{d, n} \in \mathbb{R}$ such that

$$
N(F ; B)=O_{d, \varepsilon, n}\left(B^{\theta_{d, n}+\varepsilon}\right),
$$


for any irreducible form $F \in \mathbb{Z}\left[X_{0}, \ldots, X_{n}\right]$ of degree $d \geq 2$. Then

$$
N_{Z}(B)=O_{d, \varepsilon, N}\left(B^{\theta_{d, n}+\varepsilon}\right),
$$

for any integral variety $Z \subset \mathbb{P}^{N}$ of degree $d \geq 2$ and dimension $n-1$.

In view of our remarks above concerning Conjecture 1, we may deduce from Theorem 1 that Conjecture 2 holds for arbitrary projective varieties of dimension $m \leq 3$, or for arbitrary projective quadrics. In fact it is clear that Theorem 1 has the following consequence.

Corollary 1. Conjecture 1 is equivalent to Conjecture 2.

For varieties $Z \subset \mathbb{P}^{N}$ of degree $d \geq 3$ and dimension $m \geq 4$, the best approximation to Conjecture 2 that we have is the estimate

$$
N_{Z}(B)=O_{d, \varepsilon, N}\left(B^{m+1 / d+\varepsilon}\right) .
$$

This is due to Pila [17]. The primary goal of this paper is to improve upon this estimate. In fact our method is largely inspired by the proof of (1.2), and it will be useful to recall the basic idea here. By Theorem 1 it suffices to consider the case of hypersurfaces defined by an irreducible form $F \in \overline{\mathbb{Q}}\left[X_{0}, \ldots, X_{n}\right]$ of degree $d \geq 3$.

Now for any $\nu \geq 2$, let $f \in \overline{\mathbb{Q}}\left[T_{1}, \ldots, T_{\nu}\right]$ be a non-zero polynomial of total degree $\delta$, which produces a hypersurface $f=0$ in $\mathbb{A}^{\nu}$. We define the counting function

$$
M(f ; B)=\#\left\{\mathbf{t} \in \mathbb{Z}^{\nu}: f(\mathbf{t})=0,|\mathbf{t}| \leq B\right\},
$$

for any $B \geq 1$. Then the main trick behind the proof of (1.2), as mimicked in $\S 4$ below, is the observation that

$$
N(F ; B) \leq \sum_{|b| \leq B} M\left(f_{b} ; B\right),
$$

where $f_{b}=f_{b}\left(T_{1}, \ldots, T_{n}\right)$ denotes the polynomial $F\left(b, T_{1}, \ldots, T_{n}\right)$. Pila proceeds $[17$, Theorem A] by proving the upper bound

$$
\#\left\{\mathbf{t} \in T \cap \mathbb{Z}^{\nu}:|\mathbf{t}| \leq B\right\}=O_{\delta, \varepsilon, \nu}\left(B^{\mu-1+1 / \delta+\varepsilon}\right),
$$

for any integral affine variety $T \subset \mathbb{A}^{\nu}$ of degree $\delta$ and dimension $\mu$. Such an estimate yields the corresponding bound $O_{d, \varepsilon, n}\left(B^{n-2+1 / d+\varepsilon}\right)$ for $M\left(f_{b} ; B\right)$, provided that $f_{b}$ is irreducible and has degree $d$. Upon treating the remaining degenerate cases separately, this suffices to establish (1.2).

The proof of (1.4) is argued by induction on the dimension $\mu$ of $T$. Pila starts the induction at $\mu=1$, for which he employs his earlier joint work with Bombieri [1]. Our ability to improve upon (1.2) ultimately stems from the idea of using a similar induction argument to that of Pila, but instead taking the case $\mu=2$ as the inductive base. In order to simplify the exposition of our work, it will be convenient to make the following hypothesis. 
Hypothesis (Affine surface hypothesis). Let $\varepsilon>0$ and let $\mathcal{P}$ be any set of polynomials $f \in \mathbb{Z}\left[T_{1}, T_{2}, T_{3}\right]$ of degree $\delta$. Then there exists $\alpha \in \mathbb{R}$ such that

$$
M(f ; B)=O_{\delta, \varepsilon}\left(B^{\alpha+\varepsilon}\right),
$$

for any $f \in \mathcal{P}$.

As highlighted above, the implied constant here is only permitted to depend upon $\delta$ and $\varepsilon$, and not on the individual coefficients of $f$. We shall henceforth write $\mathrm{ASH}[\alpha, \mathcal{P}]$ to denote the affine surface hypothesis holding with exponent $\alpha$ for the set of polynomials $\mathcal{P}$. Let $\mathcal{I}_{\delta}$ denote the set of irreducible polynomials in $\mathbb{Z}\left[T_{1}, T_{2}, T_{3}\right]$ of degree $\delta$. Then we have already seen via Pila's estimate (1.4) that $\mathrm{ASH}\left[1+1 / \delta, \mathcal{I}_{\delta}\right]$ holds. In fact it is easy to construct examples which show that this result is essentially best possible. Thus the irreducible polynomial $T_{1}-T_{2}^{\delta}$ has $\gg B^{1+1 / \delta}$ zeros $\mathbf{t} \in \mathbb{Z}^{3}$ with $|\mathbf{t}| \leq B$. However it is possible to take smaller exponents in the affine surface hypothesis by restricting attention to a suitable class of polynomials. This basic idea will enable us to obtain compelling evidence for Conjecture 2 . For any polynomial $g \in \mathbb{Z}\left[T_{1}, \ldots, T_{\nu}\right]$, let $\mathfrak{h}(g)$ denote the homogeneous part of $g$ of maximal degree. We let $\mathcal{J}_{\delta}$ denote the set of polynomials $f \in \mathbb{Z}\left[T_{1}, T_{2}, T_{3}\right]$ of degree $\delta$, such that $\mathfrak{h}(f)$ is irreducible. In particular it is clear that $\mathcal{J}_{\delta} \subset \mathcal{I}_{\delta}$. With this in mind, the following result will be established in $\S 6$.

Theorem 2. Let $\delta \geq 3$. Then $\operatorname{ASH}\left[\alpha, \mathcal{J}_{\delta}\right]$ holds with

$$
\alpha= \begin{cases}5 /(3 \sqrt{3})+1 / 4, & \delta=3, \\ 3 /(2 \sqrt{\delta})+1 / 3, & \delta=4 \text { or } 5, \\ 1, & \delta \geq 6 .\end{cases}
$$

We note for comparison that $\mathfrak{h}\left(T_{1}-T_{2}^{\delta}\right)$ is a product of $\delta$ linear factors, so that $T_{1}-T_{2}^{\delta} \notin \mathcal{J}_{\delta}$. In view of Theorem 2 , it seems reasonable to formulate the following conjecture.

Conjecture 3. Let $\delta \geq 2$. Then $\mathrm{ASH}\left[1, \mathcal{J}_{\delta}\right]$ holds.

We now examine how Conjecture 3 relates to Conjecture 2. The following key result will be established in $\S 4$.

Theorem 3. Let $\varepsilon>0$ and suppose that $Z \subset \mathbb{P}^{N}$ is an integral variety of degree $d \geq 2$ and dimension $m$. Then if $\mathrm{ASH}\left[\alpha, \mathcal{J}_{d}\right]$ holds we have

$$
N_{Z}(B)=O_{d, \varepsilon, N}\left(B^{m-1+\alpha+\varepsilon}\right) .
$$

In particular Conjecture 3 implies Conjecture 2.

As a direct consequence of Theorem 3, we may clearly apply Theorem 2 to deduce the following estimate. 
Corollary 2. Let $\varepsilon>0$ and suppose that $Z \subset \mathbb{P}^{N}$ is an integral variety of degree $d \geq 3$ and dimension $m$. Then we have

$$
N_{Z}(B) \ll_{d, \varepsilon, N} \begin{cases}B^{m-3 / 4+5 /(3 \sqrt{3})+\varepsilon}, & d=3, \\ B^{m-2 / 3+3 /(2 \sqrt{d})+\varepsilon}, & d=4 \text { or } 5, \\ B^{m+\varepsilon}, & d \geq 6 .\end{cases}
$$

It is worthwhile highlighting that Corollary 2 improves upon (1.2) for all values of $d \geq 3$, and plainly establishes Conjecture 2 for $d \geq 6$. We have already seen that Conjecture 2 holds for $d=2$.

We end this introduction by providing a brief summary of the contents of this paper. The following section will contain a review of some basic properties of Grassmannians, in addition to presenting a number of background estimates that will be useful to us. In $\S 3$ a birational projection argument will be used to establish Theorem 1, and in $\S 4$ we shall adapt Pila's inductive proof of (1.2) to obtain a proof of Theorem 3. The remaining sections will all be taken up with establishing Theorem 2 . Thus in $\S 5$ we shall collect together some preliminary results from algebraic geometry, before continuing with the proof of Theorem 2 proper in $\S 6$.

Acknowledgement. The authors are very grateful to the referees, for their careful reading of the text and numerous pertinent suggestions. While working on this paper, the first author was supported by EPSRC grant number GR/R93155/01.

\section{Preliminary estimates}

We begin by establishing the following simple estimate. This will be crucial to the proof of Theorem 2 in $\S 6$ below.

Lemma 1. Let $p(t)=a_{0}+a_{1} t+\cdots+a_{\delta} t^{\delta} \in \mathbb{C}[t]$ be a polynomial of degree $\delta$. Then for any $T \geq 1$ we have

$$
\#\{t \in \mathbb{Z}:|p(t)| \leq T\} \ll_{\delta} 1+\left(T /\left|a_{\delta}\right|\right)^{1 / \delta} .
$$

Proof. Our starting point in the proof of Lemma 1 is the trivial estimate

$$
\#\{t \in \mathbb{Z}:|t-\lambda| \leq L\} \ll 1+L,
$$

for any $\lambda \in \mathbb{C}$ and $L \geq 0$. Here the implied constant is independent of $\lambda$. Next we write $p(t)=a_{\delta} q(t)$, say, with $q \in \mathbb{C}[t]$ a monic polynomial. Then we may factorise $q$ as a product of linear polynomials

$$
q(t)=\prod_{i=1}^{\delta}\left(t-\lambda_{i}\right),
$$

for $\lambda_{1}, \ldots, \lambda_{\delta} \in \mathbb{C}$. Let $S(T ; p)$ denote the set of $t \in \mathbb{Z}$ for which $|p(t)| \leq T$. Then we clearly have $S(T ; p)=S\left(T /\left|a_{\delta}\right| ; q\right)$. We proceed by sorting the points 
of $S\left(T /\left|a_{\delta}\right| ; q\right)$ into sets $S_{1}, \ldots, S_{\delta}$, according to the value of $i \in\{1, \ldots, \delta\}$ for which $\left|t-\lambda_{i}\right|$ is least. But then it follows that for each $1 \leq i \leq \delta$ we have

$$
\# S_{i} \leq\left\{t \in \mathbb{Z}:\left|t-\lambda_{i}\right| \leq\left(T /\left|a_{\delta}\right|\right)^{1 / \delta}\right\} \ll 1+\left(T /\left|a_{\delta}\right|\right)^{1 / \delta},
$$

by the above. Since $S\left(T /\left|a_{\delta}\right| ; q\right)=\bigcup_{i=1}^{\delta} S_{i}$, the result follows.

Recall the notation (1.1) for the counting function attached to a variety $Z \subset \mathbb{P}^{N}$. The following "trivial" estimate is established in [4, Theorem 1].

Lemma 2. Let $Z \subset \mathbb{P}^{N}$ be a variety of degree $d$ and dimension $m$. Then we have

$$
N_{Z}(B)=O_{d, N}\left(B^{m+1}\right) .
$$

It is easy to see that Lemma 2 is best possible whenever $Z$ contains a linear subspace of dimension $m$ that is defined over $\mathbb{Q}$.

A useful consequence of Lemma 2 is that for any variety $Z \subset \mathbb{P}^{N}$ of degree $d$ and dimension $m<N$, one can always find a point $x \in \mathbb{P}^{N}(\mathbb{Q})$ such that $x \notin Z$ and $H(x)=O_{d, N}(1)$. We now discuss how this fact can be generalised somewhat. For non-negative integers $k<N$, let $\mathbb{G}(k, N)$ denote the Grassmannian which parameterises $k$-planes contained in $\mathbb{P}^{N}$. Here a $k$-plane is merely a linear subspace of $\mathbb{P}^{N}$ of dimension $k$. It is well-known that $\mathbb{G}(k, N)$ can be embedded in $\mathbb{P}^{\nu}$ via the Plücker embedding, where

$$
\nu=\left(\begin{array}{c}
N+1 \\
k+1
\end{array}\right)-1
$$

To see this explicitly, we note that since any $k$-plane $M \subset \mathbb{P}^{N}$ is spanned by $k+1$ points, we may represent $M$ as a $(k+1) \times(N+1)$ matrix $\mathbf{M}$ of rank $k+1$. The Plücker embedding is then the map which takes this matrix representation $\mathbf{M}$ to the point $\left[\operatorname{det} \mathbf{M}_{0}, \ldots, \operatorname{det} \mathbf{M}_{\nu}\right] \in \mathbb{P}^{\nu}$, where each $\mathbf{M}_{i}$ is the square matrix composed of any $k+1$ columns of $\mathbf{M}$. In fact this definition is only valid up to signs, but it is satisfactory for our purposes. One then has that $\mathbb{G}(k, N)$ is a subvariety of $\mathbb{P}^{\nu}$ of dimension $(k+1)(N-k)$. With these facts in mind we have the following result.

Lemma 3. For non-negative integers $k<N$, let $Y \subset \mathbb{G}(k, N)$ be a proper subvariety of degree $d$. Then there exists a point $y \in \mathbb{G}(k, N) \cap \mathbb{P}^{\nu}(\mathbb{Q})$ such that $y \notin Y$ and $H(y)=O_{d, N}(1)$.

Proof. Whenever $k=0$ this reduces to the statement that we can always find points of small height in $\mathbb{P}^{N}(\mathbb{Q})$ which lie off a given proper subvariety. This much has already been observed as a consequence of Lemma 2 .

For the general case $k \geq 1$, we write $\phi:\left(\mathbb{P}^{N}\right)^{k+1} \rightarrow \mathbb{P}^{\nu}$ for the rational map taking $(k+1)$-tuples of points in $\mathbb{P}^{N}$ to the point in $\mathbb{P}^{\nu}$ formed from the maximal minors of the corresponding $(k+1) \times(N+1)$ matrix. If $U \subset\left(\mathbb{P}^{N}\right)^{k+1}$ is the open subset of all $(k+1)$-tuples which span a $k$-plane, then $\phi: U \rightarrow \mathbb{P}^{\nu}$ is a morphism whose image $\phi(U)$ is the Grassmannian $\mathbb{G}(k, N) \subset \mathbb{P}^{\nu}$. We are 
interested in the inverse image $U_{Y}=\phi^{-1}(Y)$ in $U$. In particular it is clear that there exist non-zero multi-homogeneous polynomials

$$
F_{i}\left(X_{0}^{(0)}, \ldots, X_{N}^{(0)} ; \ldots ; X_{0}^{(k)}, \ldots, X_{N}^{(k)}\right), \quad(1 \leq i \leq t),
$$

say, which generate the ideal of $U_{Y}$. write

Let $\mathbf{x}^{(\ell)}=\left(X_{0}^{(\ell)}, \ldots, X_{N}^{(\ell)}\right)$ for $0 \leq \ell \leq k$. Then for any $1 \leq i \leq t$ we may

$$
F_{i}\left(\mathbf{x}^{(0)} ; \ldots ; \mathbf{x}^{(k)}\right)=\sum_{j} F_{i j}\left(\mathbf{x}^{(0)}\right) G_{i j}\left(\mathbf{x}^{(1)} ; \ldots ; \mathbf{x}^{(k)}\right)
$$

for appropriate forms $F_{i j}\left(\mathbf{x}^{(0)}\right)$ not all identically zero, and linearly independent polynomials $G_{i j}\left(\mathbf{x}^{(1)} ; \ldots ; \mathbf{x}^{(k)}\right)$. In view of Lemma 2 we may choose a point $\mathbf{a}^{(0)} \in \mathbb{Z}^{N+1}$ such that $F_{i j}\left(\mathbf{a}^{(0)}\right)$ are not all zero and $\left|\mathbf{a}^{(0)}\right|=O_{d, N}(1)$. It follows that the polynomial $F_{i}\left(\mathbf{a}^{(0)} ; \mathbf{x}^{(1)} ; \ldots ; \mathbf{x}^{(k)}\right)$ does not vanish identically. Thus we may proceed by writing

$$
F_{i}\left(\mathbf{a}^{(0)} ; \mathbf{x}^{(1)} ; \ldots ; \mathbf{x}^{(k)}\right)=\sum_{j} \tilde{F}_{i j}\left(\mathbf{a}^{(0)} ; \mathbf{x}^{(1)}\right) \tilde{G}_{i j}\left(\mathbf{x}^{(2)} ; \ldots ; \mathbf{x}^{(k)}\right),
$$

with $\tilde{F}_{i j}\left(\mathbf{a}^{(0)} ; \mathbf{x}^{(1)}\right)$ not all identically zero and $\tilde{G}_{i j}\left(\mathbf{x}^{(2)} ; \ldots ; \mathbf{x}^{(k)}\right)$ linearly independent. We then choose a point $\mathbf{a}^{(1)} \in \mathbb{Z}^{N+1}$ such that $\mathbf{a}^{(0)}$ and $\mathbf{a}^{(1)}$ are linearly independent, the numbers $F_{i j}\left(\mathbf{a}^{(0)} ; \mathbf{a}^{(1)}\right)$ are not all zero, and $\left|\mathbf{a}^{(1)}\right|=O_{d, N}(1)$. Lemma 2 ensures that this is possible. Continuing in this fashion we ultimately obtain a $(k+1)$-tuple of rational points $\left(a^{(0)}, \ldots, a^{(k)}\right) \in\left(\mathbb{P}^{N}\right)^{k+1} \backslash U_{Y}$, which span a $k$-plane and have heights

$$
H\left(a^{(0)}\right), \ldots, H\left(a^{(k)}\right)=O_{d, N}(1) .
$$

The point $y=\phi\left(a^{(0)}, \ldots, a^{(k)}\right) \in \mathbb{G}(k, N)$ is sufficient for Lemma 3 .

We end this section by recalling two further estimates that will be useful in $\S 6$. The first of these is a rather general estimate for the number of rational points of bounded height on a projective plane curve [14, Theorem 3].

Lemma 4. Let $\varepsilon>0$ and suppose that $C \subset \mathbb{P}^{2}$ is an integral curve of degree $d$. Then we have

$$
N_{C}(B)=O_{d, \varepsilon}\left(B^{2 / d+\varepsilon}\right) .
$$

Let $H(G)$ denote the maximum modulus of the coefficients of a form $G \in$ $\mathbb{Z}\left[X_{0}, \ldots, X_{n}\right]$, and say that $G$ is primitive if the highest common factor of its coefficients is 1 . The following result is established by exactly the same argument as for [14, Theorem 4].

Lemma 5. Let $G \in \mathbb{Z}\left[X_{0}, \ldots, X_{n}\right]$ be a primitive non-zero form of degree $d \geq 2$, defining a hypersurface $Z \subset \mathbb{P}^{n}$. Then either

$$
H(G) \ll B^{\theta}, \quad \theta=d\left(\begin{array}{c}
d+n \\
n
\end{array}\right),
$$

or else there exists a form $G^{\prime} \in \mathbb{Z}\left[X_{0}, \ldots, X_{n}\right]$ of degree $d$, not proportional to $G$, such that $G^{\prime}$ vanishes at each point of the set $\left\{x \in Z \cap \mathbb{P}^{n}(\mathbb{Q}): H(x) \leq B\right\}$. 


\section{Varieties in $\mathbb{P}^{N}$}

In this section we shall establish Theorem 1 . Let $Z \subset \mathbb{P}^{N}$ be an integral variety of degree $d \geq 2$ and dimension $m$. The thrust of our work will be taken up with constructing a projection $Z \rightarrow \mathbb{P}^{m+1}$, such that the image $\bar{Z} \subset \mathbb{P}^{m+1}$ is an integral variety of degree $d$ and dimension $m$. Moreover we shall want to choose our projection in such a way that we have

$$
N_{Z}(B) \leq d N_{\bar{Z}}(c B),
$$

for some $c \ll_{d, N} 1$. Once this is accomplished, the statement of Theorem 1 easily follows since now $\bar{Z} \subset \mathbb{P}^{m+1}$ is an integral hypersurface of degree $d$. We claim that such a projection always exists.

We must first deal with the possibility that $Z$ is "degenerate", by which we shall mean that $Z \subset H$ for some hyperplane $H \in \mathbb{G}(N-1, N)$. Assume that $H$ is defined by the linear equation $\sum_{i=0}^{N} a_{i} X_{i}=0$, and suppose without loss of generality that $a_{N} \neq 0$. Then the point $y=[0, \ldots, 0,1]$ is not contained in $H$ and so the projection $\pi_{y}$ from $Z$ onto the hyperplane $X_{N}=0$ is a regular map. Moreover $\pi_{y}$ is clearly birational onto the image $\bar{Z}=\pi_{y}(Z) \subset \mathbb{P}^{N-1}$, and so $\bar{Z}$ is an integral variety of degree $d$ such that $N_{Z}(B) \leq N_{\bar{Z}}(B)$. Now either $\bar{Z}$ is not degenerate, or we may repeat the argument once again. Arguing in this way it suffices to assume henceforth that $Z$ is not degenerate.

We shall need to find an $(N-m-2)$-plane $\Lambda \subset \mathbb{P}^{N}$ such that the projection $\pi_{\Lambda}: Z \rightarrow \Gamma$ from $\Lambda$ onto any $(m+1)$-plane $\Gamma$ disjoint from $\Lambda$, is birational onto the image. Moreover, we shall want $\Lambda$ to be defined over $\mathbb{Q}$ and have height $H(\Lambda) \ll_{d, N} 1$. Here we define the height of $\Lambda$ to be the standard multiplicative height of its coordinates in $\mathbb{G}(N-m-2, N)$, under the Plücker embedding. Now it is well-known that for a generic $\Lambda \in \mathbb{G}(N-m-2, N)$ the projection described above is birational. Hence the main work comes from showing that one can always find such a linear space which is defined over $\mathbb{Q}$ and which has small height. To do so we shall need to establish the following preliminary result.

Lemma 6. Let $Z \subset \mathbb{P}^{N}$ be an integral variety of degree $d$ and dimension $m$. Let

$$
Y=\left\{\Lambda \in \mathbb{G}(N-m-2, N): \operatorname{dim} S_{\Lambda, Z} \geq m\right\},
$$

where $S_{\Lambda, Z}$ denotes the set of $M \in \mathbb{G}(N-m-1, N)$ such that $\Lambda \subset M$ and $M$ intersects $Z$ in at least two (possibly coincident) points. Then $Y$ is a proper subvariety of $\mathbb{G}(N-m-2, N)$ of degree $O_{d, N}(1)$.

Proof. It is well known that varieties in $\mathbb{P}^{N}$ of degree $d$ and dimension $m$ can be parameterised by a quasi-projective variety $\mathcal{C}_{d, m}\left(\mathbb{P}^{N}\right) \subset \mathbb{P} U$, where $U$ is the vector space of multi-homogeneous polynomials of multi-degree $(d, \ldots, d)$ in $m+1$ sets of $N+1$ variables. This is the open Chow variety (see [11, Theorem 21.2], for example) of varieties of degree $d$ and dimension $m$ in $\mathbb{P}^{N}$. If $V \subset \mathbb{P}^{N}$ is any variety of degree $d$ and dimension $m$, we let $\left[c_{V}\right]$ denote the corresponding 
Chow point in $\mathcal{C}_{d, m}\left(\mathbb{P}^{N}\right)$. We shall also denote by $F_{d, m}$ the universal family of all $m$-dimensional subvarieties of degree $d$ in $\mathbb{P}^{N}$. It is the closed subvariety $F_{d, m} \subset \mathcal{C}_{d, m}\left(\mathbb{P}^{N}\right) \times \mathbb{P}^{N}$ of points $\left(\left[c_{V}\right], x\right) \in \mathcal{C}_{d, m}\left(\mathbb{P}^{N}\right) \times \mathbb{P}^{N}$ such that $x \in V$. Much as we have followed the convention of identifying linear spaces in $\mathbb{P}^{N}$ with the corresponding Plücker point in the Grassmannian, we shall frequently write $V$ to denote the Chow point $\left[c_{V}\right]$.

Write $\mathcal{C}=\mathcal{C}_{d, m}\left(\mathbb{P}^{N}\right), G_{1}=\mathbb{G}(N-m-2, N)$ and $G_{2}=\mathbb{G}(N-m-1, N)$ for convenience. We proceed by considering the incidence correspondence

$$
I=\left\{(\Lambda, M, V) \in G_{1} \times G_{2} \times \mathcal{C}: \Lambda \subset M, \#(M \cap V) \geq 2\right\} .
$$

Here the condition \# $(M \cap V) \geq 2$ asserts that $M$ intersects $V$ in at least two (possibly coincident) points. We formally define $\#(M \cap V)$ to be infinity if the intersection of $M$ and $V$ contains a component of dimension $\geq 1$. Let $I_{1} \subset G_{1} \times G_{2}$ be the set of $(\Lambda, M) \in G_{1} \times G_{2}$ such that $\Lambda \subset M$, and let $I_{2} \subset G_{2} \times \mathcal{C}$ be the set of $(M, V) \in G_{2} \times \mathcal{C}$ such that $\#(M \cap V) \geq 2$. Then if $\phi_{1}$ denotes the projection $G_{1} \times G_{2} \times \mathcal{C} \rightarrow G_{1} \times G_{2}$, and $\phi_{2}$ the projection $G_{1} \times G_{2} \times \mathcal{C} \rightarrow G_{2} \times \mathcal{C}$, then it is plain that $I=\phi_{1}^{-1}\left(I_{1}\right) \cap \phi_{2}^{-1}\left(I_{2}\right)$. Thus in order to show that $I$ is closed in the Zariski topology, it suffices to show that $I_{1}$ and $I_{2}$ are both closed sets. It is well-known that $I_{1}$ is closed (see [9, Proposition I.9.9.3], for example). To see that $I_{2}$ is closed in $G_{2} \times \mathcal{C}$ we show that the intersection cardinality of $M$ and $V$ is an upper semicontinuous function. Let $\Omega_{1}$ be the set of $(M, V) \in G_{2} \times \mathcal{C}$ such that $M \cap V$ contains a component of dimension $\geq 1$, and let $\Omega_{2}=I_{2} \backslash \Omega_{1}$. It is clear that $\Omega_{1}$ is closed, and so it remains to show that $\#(M \cap V) \geq n$ gives a closed condition on $\Omega_{2}$, for any positive integer $n$. To do so we apply [12, Excercise II.5.8(a)] to the restriction of the coherent sheaf $\phi_{*} \mathcal{O}_{Z}$ to $\Omega_{2}$ for the projection $\phi: Z \rightarrow G_{2} \times \mathcal{C}$ from the closed subset $Z \subseteq G_{2} \times F_{d, n}$ of all $(M, V, x) \in G_{2} \times F_{d, n}$ such that $x \in M$.

Let $\pi: I \rightarrow G_{1} \times \mathcal{C}$ be the projection map, and let $W \subset G_{1} \times \mathcal{C}$ denote the subset over which the fibre of $\pi$ has dimension at least $m$. Then $W$ is a closed subvariety of $G_{1} \times \mathcal{C}$, by the upper semicontinuity of fibre dimension [8, Corollaire 13.1.5]. Let $\left[c_{Z}\right] \in \mathcal{C}$ be the Chow point corresponding to the variety $Z$. Then if $\rho: W \rightarrow \mathcal{C}$ denotes the projection onto the second factor, we consider the fibre

$$
\rho^{-1}\left(\left[c_{Z}\right]\right)=\left\{\Lambda \in G_{1}:(\Lambda, Z) \in W\right\} .
$$

It follows that $\rho^{-1}\left(\left[c_{Z}\right]\right)$ is a projective subvariety of $G_{1}=\mathbb{G}(N-m-2, N)$, whose degree can be bounded in terms of $d$ and $N$ alone. To see this we recall the embedding $G_{1} \times \mathcal{C} \subset \mathbb{P}^{r} \times \mathbb{P} U$, with $r=\left(\begin{array}{c}N+1 \\ N-m-1\end{array}\right)-1$, so that $W \subset \mathbb{P}^{r} \times \mathbb{P} U$ is cut out by $O_{d, N}(1)$ multi-homogeneous polynomials of multi-degree $O_{d, N}(1)$. The claim then follows on specialising at $\left[c_{Z}\right]$, and applying Bézout's theorem (in the form given by $[7$, Theorem 8.4 .6$])$. Finally we note that $Y=\rho^{-1}\left(\left[c_{Z}\right]\right.$ ) in the statement of the lemma.

In order to complete the proof of Lemma 6 we need to show that $Y$ is a proper subvariety of $G_{1}$. As in the statement of the lemma let $S_{\Lambda, Z}$ denote the set of $(N-m-1)$-planes containing $\Lambda$ and meeting $Z$ in at least two points, for any $\Lambda \in G_{1}$. Now let $\Lambda \in G_{1}$ be such that $\Lambda \cap Z$ is empty, and let $\rho_{\Lambda}: Z \rightarrow G_{2}$ 
be the map given by sending a point $x \in Z$ to the projective linear subspace $\langle x, \Lambda\rangle$ spanned by $x$ and $\Lambda$. Then $\rho_{\Lambda}$ is birational onto its image if and only if $\operatorname{dim} S_{\Lambda, Z}<m$. This follows on combining the observation that $S_{\Lambda, Z} \subset \rho_{\Lambda}(Z)$ is the proper closed subset of $M \in \rho_{\Lambda}(Z)$ such that $\rho_{\Lambda}^{-1}(M)$ consists of more than one point, with the fact that $\rho_{\Lambda}(Z)$ is integral and has dimension $m$. Now let $\Lambda \in G_{1}$ be generic. Then it is well known that the map $\rho_{\Lambda}$ is birational onto its image [11, Exercise 11.23]. We therefore conclude that $\Lambda \notin Y$, which thereby completes the proof of Lemma 6.

We now proceed with the proof of Theorem 1. Let $Y^{\prime}$ denote the set of $\Lambda \in$ $\mathbb{G}(N-m-2, N)$ which meet $Z$. Then $Y^{\prime}$ is a proper subvariety of $\mathbb{G}(N-m-2, N)$ and has degree $O_{d, N}(1)$. In view of Lemma 3, we may therefore conclude from Lemma 6 that there exists an $(N-m-2)$-plane $\Lambda \subset \mathbb{P}^{N}$ such that $\Lambda$ is defined over $\mathbb{Q}$ and has height $H(\Lambda) \ll_{d, N} 1$, and such that $\Lambda \notin Y \cup Y^{\prime}$. In particular $\Lambda \cap Z$ is empty and the projection $\rho_{\Lambda}$ from $\Lambda$ is birational onto its image. Below we shall select a certain linear space $\Gamma \in \mathbb{G}(m+1, N)$ which does not meet $\Lambda$, and which is defined over $\mathbb{Q}$ and has height $H(\Gamma) \ll_{d, N} 1$. For the moment, let $\Gamma \in \mathbb{G}(m+1, N)$ be any $(m+1)$-plane that does not meet $\Lambda$, and let

$$
\pi_{\Lambda}: Z \rightarrow \Gamma
$$

be the projection of $Z$ from $\Lambda$ onto $\Gamma$. Then by construction $\pi_{\Lambda}$ is a regular map that is birational onto its image, and we may therefore conclude that the image $\bar{Z}=\pi_{\Lambda}(Z) \subset \Gamma$ is an integral variety of dimension $m$ and degree $d$. Finally we note that the fibre over any point of $\bar{Z}$ contains at most $d$ points.

Now suppose that $\Lambda$ is equal to the linear span of $N-m-1$ points

$$
\left[\mathbf{h}_{1}\right], \ldots,\left[\mathbf{h}_{N-m-1}\right] \in \mathbb{P}^{N},
$$

for vectors $\mathbf{h}_{1}, \ldots, \mathbf{h}_{N-m-1} \in \mathbb{Z}^{N+1}$ of modulus $O_{d, N}(1)$. Then by Lemma 3 we may select a vector $\mathbf{g}_{1} \in \mathbb{Z}^{N+1}$ of modulus $O_{d, N}(1)$ such that $\mathbf{g}_{1} \cdot \mathbf{h}_{j}=0$ for $2 \leq j \leq N-m-1$, and $\mathbf{g}_{1} \cdot \mathbf{h}_{1} \neq 0$. Similarly, for $2 \leq i \leq N-m-1$ we may find vectors $\mathbf{g}_{i} \in \mathbb{Z}^{N+1}$ of modulus $O_{d, N}(1)$ such that $\mathbf{g}_{i} \cdot \mathbf{h}_{j}=0$ for $i \neq j$ and $\mathbf{g}_{i} \cdot \mathbf{h}_{i} \neq 0$. These vectors are clearly linearly independent, and define the $(m+1)$-plane

$$
\Gamma: \quad \mathbf{g}_{1} \cdot \mathbf{x}=\cdots=\mathbf{g}_{N-m-1} \cdot \mathbf{x}=0 .
$$

By construction $\Lambda \cap \Gamma$ is empty. We may now explicitly write our projection in the form

$$
\pi_{\Lambda}(x)=\left[\mathbf{x}-\frac{\left(\mathbf{g}_{1} \cdot \mathbf{x}\right)}{\left(\mathbf{g}_{1} \cdot \mathbf{h}_{1}\right)} \mathbf{h}_{1}-\cdots-\frac{\left(\mathbf{g}_{N-m-1} \cdot \mathbf{x}\right)}{\left(\mathbf{g}_{N-m-1} \cdot \mathbf{h}_{N-m-1}\right)} \mathbf{h}_{N-m-1}\right],
$$

for any $x=[\mathbf{x}] \in Z$. Let $\lambda=\prod_{i} \mathbf{g}_{i} \cdot \mathbf{h}_{i}$ and let $\lambda_{j}=\prod_{i \neq j} \mathbf{g}_{i} \cdot \mathbf{h}_{i}$. Then since $\left|\mathbf{g}_{i} \cdot \mathbf{x}\right| \leq(N+1)\left|\mathbf{g}_{i}\right||\mathbf{x}|$ for $1 \leq i \leq N-m-1$, we deduce that $H\left(\pi_{\Lambda}(x)\right) \leq c H(x)$ with

$$
c=|\lambda|+(N+1) \sum_{i=1}^{N-m-1}\left|\lambda_{i}\right| H\left(\left[\mathbf{g}_{i}\right]\right) H\left(\left[\mathbf{h}_{i}\right]\right) \ll_{d, N} 1 .
$$

It therefore follows that (3.1) holds, as claimed, with $\bar{Z}=\pi_{\Lambda}(Z) \subset \mathbb{P}^{m+1}$. This completes the proof of Theorem 1 . 


\section{Affine hypersurfaces}

In this section we shall establish Theorem 3. The main step in the proof will involve using the affine surface hypothesis to deduce a corresponding bound for affine hypersurfaces. In view of Theorem 1 it will suffice to establish the estimate

$$
N(F ; B)=O_{d, \varepsilon, n}\left(B^{n-2+\alpha+\varepsilon}\right),
$$

for any irreducible form $F \in \mathbb{Z}\left[X_{0}, \ldots, X_{n}\right]$ of degree $d \geq 2$, provided that ASH $\left[\alpha, \mathcal{J}_{d}\right]$ holds. Let $X \subset \mathbb{P}^{n}$ denote the hypersurface defined by the equation $F=0$. We shall need some control over the hyperplanes $H \in \mathbb{G}(n-1, n)=\mathbb{P}^{n *}$ for which $X \cap H$ is not integral. For this we shall apply the following well-known result (see [3, Lemma 2.2.1], for example).

Lemma 7. Let $n \geq 3$ and let $X \subset \mathbb{P}^{n}$ be an integral hypersurface of degree $d$. Then there exists a non-zero form $E \in \mathbb{Z}\left[X_{0}, \ldots, X_{n}\right]$ of degree $O_{d, n}(1)$, such that $E(\mathbf{a})=0$ whenever the hyperplane

$$
H_{\mathbf{a}}: \quad a_{0} X_{0}+\cdots+a_{n} X_{n}=0
$$

produces a hyperplane section $X \cap H_{\mathbf{a}}$ that is not integral.

Lemma 7 may be viewed as an explicit statement of the basic geometric fact that the generic hyperplane section of an integral variety is integral. Using Lemma 2 and Lemma 7 we may find a vector $\mathbf{a} \in \mathbb{Z}^{n+1}$ such that $|\mathbf{a}| \ll_{d, n} 1$ and $X \cap H_{\mathbf{a}}$ is integral. After a suitable change of coordinates we may assume henceforth that $H_{\mathbf{a}}$ is the hyperplane $X_{0}=0$, and hence that the form $F\left(0, X_{1}, \ldots, X_{n}\right)$ is irreducible.

For any $b \in \mathbb{Z}$ we define the polynomial $f_{b}\left(T_{1}, \ldots, T_{n}\right)=F\left(b, T_{1}, \ldots, T_{n}\right)$. Then $f_{b} \in \mathbb{Z}\left[T_{1}, \ldots, T_{n}\right]$ is a polynomial of degree $d$ such that $\mathfrak{h}\left(f_{b}\right)$ is irreducible, so that $f_{b} \in \mathcal{J}_{d}$. Moreover (1.3) implies that

$$
N(F ; B) \leq \sum_{|b| \leq B} M\left(f_{b} ; B\right) .
$$

In order to complete the proof of Theorem 3 it is therefore sufficient to establish the following result.

Lemma 8. Let $\varepsilon>0$ and suppose that $\mathrm{ASH}\left[\alpha, \mathcal{J}_{\delta}\right]$ holds. For any $\nu \geq 3$ let $f \in \mathbb{Z}\left[T_{1}, \ldots, T_{\nu}\right]$ be a polynomial of degree $\delta$ such that $\mathfrak{h}(f)$ is irreducible. Then we have

$$
M(f ; B) \ll_{\delta, \varepsilon, \nu} B^{\nu-3+\alpha+\varepsilon} .
$$

Proof. The proof of Lemma 8 will involve taking repeated hyperplane sections of the affine hypersurface $f=0$. We shall argue by induction on $\nu$. The case $\nu=3$ is satisfactory by the assumption that $\operatorname{ASH}\left[\alpha, \mathcal{J}_{\delta}\right]$ holds. For general $\nu \geq 4$ we write $f_{0}=\mathfrak{h}(f)$, so that $f_{0}\left(T_{1}, \ldots, T_{\nu}\right)$ is an irreducible form of degree $\delta$ by assumption. Employing Lemma 2 and Lemma 7 we may therefore assume, possibly after a change of variables, that the hyperplane section $f_{0}=$ 
$T_{\nu}=0$ produces an integral hypersurface. That is, we may assume that the homogeneous part of the polynomial $f_{c}=f\left(T_{1}, \ldots, T_{\nu-1}, c\right)$ is irreducible for any $c \in \mathbb{Z}$. Thus we obtain the bound

$$
M(f ; B) \leq \sum_{|c| \leq B} M\left(f_{c} ; B\right) \ll_{\delta, \varepsilon, \nu} B^{\nu-3+\alpha+\varepsilon},
$$

by the induction hypothesis. This completes the proof of Lemma 8 .

\section{Geometry of surfaces}

The remainder of the paper will be taken up with the proof of Theorem 2 . In this section we collect together some of the geometric results that will be necessary to us, throughout which $X \subset \mathbb{P}^{3}$ will always denote a surface of degree $d \geq 3$. Let $H \in \mathbb{P}^{3^{*}}$ be a plane such that $X \cap H$ is integral, where $\mathbb{P}^{3^{*}}=\mathbb{G}(2,3)$ as above, and let $C \subset X$ be an integral curve of degree $e<d$. In particular it follows that $X$ is integral and that $C$ is not contained in the plane section $X \cap H$. For given $y \in X \cap H$, we shall begin by investigating the integral curves $C \subset X$ of degree $e<d$ that have intersection multiplicity $e$ with $H$ at $y$.

Lemma 9. Let $e<d$ and let $H \in \mathbb{P}^{3^{*}}$ be a plane such that $X \cap H$ is integral. Then for any $y \in X \cap H$ there are $O_{d}(1)$ integral curves $C \subset X$ of degree e such that $C$ has intersection multiplicity $e$ with $H$ at $y$.

Proof. Let $\mathcal{P}$ be the set of all Hilbert polynomials of integral space curves of degree $e$. Then Castelnuovo's inequality implies that $\mathcal{P}$ is finite. Let $\mathcal{H}$ be the Hilbert scheme of all closed subschemes of $\mathbb{P}^{3}$ with Hilbert polynomials in $\mathcal{P}$, and let $\mathcal{H}^{\prime}$ be the Hilbert scheme of all closed surfaces $X \subset \mathbb{P}^{3}$ of degree $d$. We shall also need to introduce the universal family $\mathcal{F} \subset \mathbb{P}^{3} \times \mathcal{H}$ of all closed subschemes of $\mathbb{P}^{3}$ with Hilbert polynomials in $\mathcal{P}$, and the open subscheme $\mathcal{H}_{0}$ of $\mathcal{H}$ representing curves not in $H$.

For any pair $P=(C, y) \in \mathcal{H}_{0} \times H$, let $i_{y}(C \cap H)$ denote the intersection multiplicity of $C$ and $H$ at $y$. Also, let $\mathcal{G} \subset \mathbb{P}^{3} \times \mathcal{H} \times H$ be the scheme-theoretic intersection of $\mathcal{F} \times H \subset \mathbb{P}^{3} \times \mathcal{H} \times H$ with the inverse image of the diagonal $H^{\Delta} \subset \mathbb{P}^{3} \times H$ under the projection $\mathbb{P}^{3} \times \mathcal{H} \times H \rightarrow \mathbb{P}^{3} \times H$. Then $i_{y}(C \cap H)$ can be interpreted as $\operatorname{dim}_{k(P)} \phi_{*} \mathcal{O}_{\mathcal{G}} \times k(P)$ for the projection $\phi: \mathcal{G} \rightarrow \mathcal{H} \times H$. Hence $i_{y}(C \cap H)$ is an upper semicontinuous function on $\mathcal{H}_{0} \times H$ by [12, Exercise II.5.8]. We can extend it to an upper semicontinuous function on $\mathcal{H} \times H$ by formally defining $i_{y}(C \cap H)$ to be infinity if $C \subset H$.

We shall work with the closed subscheme $W \subset \mathcal{H} \times \mathcal{H}^{\prime}$ of pairs $(C, X) \in$ $\mathcal{H} \times \mathcal{H}^{\prime}$ such that $C$ is a closed subscheme of $X$ (compare [5, pp. 265-266], for example). Let $Z \subset W \times H$ be the closed subscheme of triples $(C, X, y)$ such that $i_{y}(C \cap H) \geq e$. If we choose embeddings $\mathcal{H} \subset \mathbb{P}^{N}$ and $\mathcal{H}^{\prime} \subset \mathbb{P}^{N^{\prime}}$ into projective space, then $Z$ is a closed subscheme of $\mathbb{P}^{N} \times \mathbb{P}^{N^{\prime}} \times H$ defined by finitely many trihomogeneous polynomials. Therefore the fibre $Z_{X, y}$ of the projection from $Z \subset \mathcal{H} \times \mathcal{H}^{\prime} \times H$ to $\mathcal{H}^{\prime} \times H$ is a closed subscheme of $\mathbb{P}^{N}$ 
defined by $O_{d}(1)$ forms of degree $O_{d}(1)$ in $N+1$ variables. An application of Bézout's theorem (in the form given by [7, Theorem 8.4.6]) therefore reveals that $\# Z_{X, y}=O_{d}(1)$, provided that $Z_{X, y}$ is zero-dimensional. This scheme parameterises all closed curves $C \subset X$ of degree $e$ such that $C$ has Hilbert polynomial in $\mathcal{P}$ and $i_{y}(C \cap H) \geq e$. To complete the proof of Lemma 9, it is therefore sufficient to prove that $Z_{X, y}$ is zero-dimensional or empty, whenever $X \cap H$ is integral and $y \in X \cap H$.

We prove this by contradiction. Let $\mathcal{F}_{X, y} \subset X \times Z_{X, y}$ be the family of closed subschemes of $X$, obtained by pulling back the universal family $\mathcal{F} \subset \mathbb{P}^{3} \times \mathcal{H}$ along the projection of $Z \subset \mathcal{H} \times \mathcal{H}^{\prime} \times H$ to $\mathcal{H}$. Let $Y$ be the image of $\mathcal{F}_{X, y}$ under the projection onto $X$. Then $Y$ is a closed subscheme of $X$, and must have dimension at least 2 if $\operatorname{dim} Z_{X, y} \geq 1$. Since $Y \subseteq X$, and $X$ is integral, we must therefore conclude that $Y=X$. Suppose now that $x \in X \cap H$ is any point distinct from $y$. Then there exists a curve $C \subset X$ of degree $e$, such that $x \in C$ and $C$ has intersection multiplicity $e$ with $H$ at $y$. But then it follows from Bézout's theorem that $C \cap H$ contains a component of dimension 1, since both $x$ and the $e$-fold point $y$ lie on $C \cap H$. This plainly contradicts the assumption that $X \cap H$ is integral, and so completes the proof of Lemma 9.

We shall apply Lemma 9 only in the special cases $e=1$ and $e=2$. Suppose that $H \in \mathbb{P}^{3^{*}}$ is such that $X \cap H$ is integral, and let $y \in X \cap H$. Then in the former case it follows from Lemma 9 that

$$
\#\left\{\Lambda \in F_{1}(X): y \in \Lambda\right\}=O_{d}(1),
$$

where $F_{1}(X)$ is the Fano variety $\{\Lambda \in \mathbb{G}(1,3): \Lambda \subset X\}$ of lines contained in $X$. In the latter case it allows us to conclude that there are only $O_{d}(1)$ conics contained in $X$ that are tangent to $H$ at $y$.

As above let $X \subset \mathbb{P}^{3}$ be a projective surface. For any non-singular point $x \in X$, we let $\mathbb{T}_{x}(X)$ denote the tangent plane to $X$ at $x$. The remainder of this section is concerned with the set of non-singular points $x \in X$ which have multiplicity at most 2 on the intersection $X \cap \mathbb{T}_{x}(X)$. We begin by establishing the following result.

Lemma 10. Suppose that $X \subset \mathbb{P}^{3}$ is an integral surface which is not a plane. Then there exists a non-singular point $x \in X$ such that $x$ has multiplicity 2 on the intersection $X \cap \mathbb{T}_{x}(X)$.

Proof. Since $X$ is integral, Bertini's theorem ensures the existence of a plane $H \in \mathbb{P}^{3 *}$ such that the intersection $Y=X \cap H$ is integral. Now it is well known that given any integral plane curve of degree exceeding 1 , the Hessian of the curve does not vanish identically on the curve (see Fischer [6, §4.5], for example). Hence we may find a non-singular point $x \in Y$ which is not an inflection point. But then it follows that $x$ is of multiplicity 2 on the intersection $X \cap \mathbb{T}_{x}(X) \cap H$, and thus also on the intersection $X \cap \mathbb{T}_{x}(X)$.

The following result allows us to conclude that the set of non-singular points $x \in X$ which have multiplicity at most 2 on the intersection $X \cap \mathbb{T}_{x}(X)$, is open in the Zariski topology. 
Lemma 11. Let d be a positive integer. Then there exists a finite set of universal bihomogeneous polynomials

$$
\Phi_{0}\left(a_{\mathbf{e}} ; X_{0}, X_{1}, X_{2}, X_{3}\right), \ldots, \Phi_{t}\left(a_{\mathbf{e}} ; X_{0}, X_{1}, X_{2}, X_{3}\right),
$$

with coefficients in $\mathbb{Z}$, such that the following holds for any field $K$. A point $x=\left[x_{0}, x_{1}, x_{2}, x_{3}\right]$ on the surface defined by the form

$$
F=\sum_{\begin{array}{c}
e_{0}, \ldots, e_{3} \geq 0 \\
e_{0}+e_{1}+e_{2}+e_{3}=d
\end{array}} a_{\mathbf{e}} X_{0}^{e_{0}} X_{1}^{e_{1}} X_{2}^{e_{2}} X_{3}^{e_{3}} \in K\left[X_{0}, X_{1}, X_{2}, X_{3}\right],
$$

is a non-singular point of multiplicity at most 2 on the section with the tangent plane at $x$ if and only if $\Phi_{i}\left(a_{\mathbf{e}} ; x_{0}, x_{1}, x_{2}, x_{3}\right) \neq 0$ for some $0 \leq i \leq t$.

Proof. Let $X \subset \mathbb{P}^{3}$ be the surface defined by the form (5.1), and let $x=[\mathbf{x}] \in X$ be a non-singular point. Then there exists a linear form $L$ and a quadratic form $Q$, such that

$$
F(\mathbf{x}+\lambda \mathbf{y}) \equiv L(\mathbf{y}) \lambda+Q(\mathbf{y}) \lambda^{2}\left(\bmod \lambda^{3}\right) .
$$

In fact, if we write $\mathbf{g}=\nabla F(\mathbf{x})$ and $\mathbf{M}=\mathbf{M}(\mathbf{x})$ for the the matrix of second derivatives of $F$ at $\mathbf{x}$, then $L(\mathbf{y})=\mathbf{g} \cdot \mathbf{y}$ and $2 Q(\mathbf{y})=\mathbf{y}^{T} \mathbf{M y}$. Moreover it is plain that $\sum_{i=0}^{3} g_{i} X_{i}=0$ is the equation for the tangent plane $\mathbb{T}_{x}(X)$ at $x$. It follows that $x=[\mathbf{x}]$ has multiplicity at most 2 on the tangent plane section $X \cap \mathbb{T}_{x}(X)$ if and only if $Q(\mathbf{y}) \neq 0$ for some vector $\mathbf{y}$ such that $\mathbf{g} . \mathbf{y}=0$. But $\mathbb{T}_{x}(X)$ is plainly spanned by the vectors

$$
\begin{aligned}
& \mathbf{y}_{1}=\left(g_{1},-g_{0}, 0,0\right), \quad \mathbf{y}_{2}=\left(g_{2}, 0,-g_{0}, 0\right), \quad \mathbf{y}_{3}=\left(g_{3}, 0,0,-g_{0}\right), \\
& \mathbf{y}_{4}=\left(0, g_{2},-g_{1}, 0\right), \quad \mathbf{y}_{5}=\left(0, g_{3}, 0,-g_{1}\right), \quad \mathbf{y}_{6}=\left(0,0, g_{3},-g_{2}\right),
\end{aligned}
$$

and so $x \in X$ is a non-singular point of multiplicity at most 2 on the tangent plane section if and only if $\mathbf{g} \neq \mathbf{0}$ and $Q\left(\mathbf{y}_{i}\right) \neq 0$ for some $1 \leq i \leq 6$. This therefore establishes the existence of the bihomogeneous polynomials

$$
\Phi_{0}\left(a_{\mathbf{e}} ; X_{0}, X_{1}, X_{2}, X_{3}\right), \ldots, \Phi_{t}\left(a_{\mathbf{e}} ; X_{0}, X_{1}, X_{2}, X_{3}\right),
$$

that appear in the statement of the lemma.

Suppose that $X \subset \mathbb{P}^{3}$ is an integral surface defined over a field $K$. We henceforth let $U$ denote the set of non-singular points on $X$ which have multiplicity at most 2 on the tangent plane section at the point. On combining Lemma 10 with Lemma 11 it follows that $U$ is a non-empty open subset of $X$. Thus the complement of $U$ in $X$ consists of finitely many integral components of dimension at most 1. Furthermore, we may apply Bézout's theorem (in the form given by Fulton [7, Theorem 8.4.6]) to deduce that the sum of the degrees of these components is bounded in terms of the maximal degree of the specialisation of the universal polynomials $\Phi_{0}, \ldots, \Phi_{t}$ at $X$. Thus the complement of $U$ in $X$ consists of $O_{d}(1)$ integral components of degree $O_{d}(1)$. 


\section{Affine surfaces}

In order to prove Theorem 2 it will be convenient to work with a projective model for the affine surface $f=0$. Thus let $X \subset \mathbb{P}^{3}$ be the surface defined by the form

$$
F\left(X_{0}, X_{1}, X_{2}, X_{3}\right)=X_{0}^{\delta} f\left(X_{1} / X_{0}, X_{2} / X_{0}, X_{3} / X_{0}\right),
$$

of degree $\delta$. We may assume that $F$ has integer coefficients whose highest common factor is 1 . Moreover it is clear that $\mathfrak{h}(f)$ is irreducible if and only if $X \cap H_{\infty}$ is integral, where $H_{\infty}$ denotes the plane $X_{0}=0$.

As indicated above we shall represent elements of $\mathbb{P}^{n}(\mathbb{Q})$ by $(n+1)$-tuples $\mathbf{x}=\left(x_{0}, \ldots, x_{n}\right) \in \mathbb{Z}^{n+1}$ such that h.c.f. $\left(x_{0}, \ldots, x_{n}\right)=1$, for any $n \geq 2$. The pairs $[\mathbf{x}]= \pm \mathbf{x}$ of such $(n+1)$-tuples corresponds to integral points on the scheme $\mathbb{P}_{\mathbb{Z}}^{n}$. We shall therefore write $[\mathbf{x}] \in \mathbb{P}^{n}(\mathbb{Z})$ to express that $\mathbf{x} \in \mathbb{Z}^{n+1}$ and h.c.f. $\left(x_{0}, \ldots, x_{n}\right)=1$. This choice of notation is motivated by the fact that we shall constantly deal with integral points on the affine cone over $\mathbb{Z}$, rather than over $\mathbb{Q}$. With this in mind we define the counting function

$$
N^{\mathrm{aff}}(\Sigma ; B)=\#\left\{\left[1, x_{1}, x_{2}, x_{3}\right] \in \Sigma \cap \mathbb{P}^{3}(\mathbb{Z}): H\left(\left[1, x_{1}, x_{2}, x_{3}\right]\right) \leq B\right\},
$$

for any locally closed subset $\Sigma \subseteq X$ defined over $\overline{\mathbb{Q}}$.

Let $F \in \mathbb{Z}\left[X_{0}, X_{1}, X_{2}, X_{3}\right]$ be a primitive form of degree $d \geq 3$, defining a surface $X \subset \mathbb{P}^{3}$ such that $X \cap H_{\infty}$ is integral. It is now clear that in order to establish Theorem 2 it will suffice to establish the estimate

$$
N^{\text {aff }}(X ; B) \ll_{d, \varepsilon} \begin{cases}B^{5 /(3 \sqrt{3})+1 / 4+\varepsilon}, & d=3, \\ B^{1+\varepsilon}+B^{3 /(2 \sqrt{d})+1 / 3+\varepsilon}, & d \geq 4,\end{cases}
$$

for any $\varepsilon>0$. For any prime $p$ we shall write $X_{p}$ for the surface defined over $\mathbb{F}_{p}$ obtained by reducing the coefficients of $F$ modulo $p$, and we denote the set $X_{p} \cap \mathbb{P}^{3}\left(\mathbb{F}_{p}\right)$ by $X_{p}\left(\mathbb{F}_{p}\right)$. Note that, as a scheme, $X_{p}$ is not necessarily integral. It will be convenient to define the set

$$
S(\Sigma ; B)=\left\{\left[1, x_{1}, x_{2}, x_{3}\right] \in \Sigma \cap \mathbb{P}^{3}(\mathbb{Z}): H\left(\left[1, x_{1}, x_{2}, x_{3}\right]\right) \leq B\right\},
$$

for any locally closed subset $\Sigma \subseteq X$ defined over $\overline{\mathbb{Q}}$. In particular we have $N^{\text {aff }}(\Sigma ; B)=\# S(\Sigma ; B)$, by $(6.2)$. Now let $\pi=\left[1, \pi_{1}, \pi_{2}, \pi_{3}\right] \in X_{p}\left(\mathbb{F}_{p}\right)$, where $\pi_{1}, \pi_{2}, \pi_{3}$ are always assumed to be in $\mathbb{F}_{p}$. We also define the set

$$
S_{p}(\Sigma ; B, \pi)=\left\{\left[1, x_{1}, x_{2}, x_{3}\right] \in \Sigma \cap \mathbb{P}^{3}(\mathbb{Z}): \begin{array}{l}
H\left(\left[1, x_{1}, x_{2}, x_{3}\right]\right) \leq B, \\
x_{i} \equiv \pi_{i}(\bmod p),(1 \leq i \leq 3)
\end{array}\right\},
$$

for any locally closed subset $\Sigma \subseteq X$ defined over $\overline{\mathbb{Q}}$. It is clear that $S_{p}(\Sigma ; B, \pi)$ may be empty if there are no points on $\Sigma$ which specialise to $\pi$ on $X_{p}$.

Recall the definition of the non-empty open subset $U \subset X$, introduced at the close of $\S 5$. For any prime $p$ we shall define $U_{p}$ to be the corresponding open set of non-singular points on $X_{p}$ which have multiplicity at most 2 on the tangent plane section at the point. Our main tool in the proof of Theorem 2 will be the following adaption of a result due to the second author [14, Theorem 14]. 
Lemma 12. Let $\varepsilon>0$ and let $X \subset \mathbb{P}^{3}$ be an integral surface, defined by a primitive form $F \in \mathbb{Z}\left[X_{0}, X_{1}, X_{2}, X_{3}\right]$ of degree $d \geq 3$ such that

$$
\log H(F)=O_{d}(\log B) .
$$

Then there exists a set $\Pi$ of $O_{d, \varepsilon}(1)$ primes $p$, with

$$
B^{1 / \sqrt{d}+\varepsilon} \ll_{d, \varepsilon} p \ll_{d, \varepsilon} B^{1 / \sqrt{d}+\varepsilon},
$$

such that the following holds. For each $\pi=\left[1, \pi_{1}, \pi_{2}, \pi_{3}\right] \in U_{p}\left(\mathbb{F}_{p}\right)$, there exists a form $G_{\pi} \in \mathbb{Z}\left[X_{0}, X_{1}, X_{2}, X_{3}\right]$ of degree $O_{d, \varepsilon}(1)$ which is not divisible by $F$, such that

$$
S(U ; B)=\bigcup_{p \in \Pi} \bigcup_{\pi \in U_{p}\left(\mathbb{F}_{p}\right)}\left\{\left[1, x_{1}, x_{2}, x_{3}\right] \in S_{p}(U ; B, \pi): G_{\pi}\left(1, x_{1}, x_{2}, x_{3}\right)=0\right\} .
$$

Proof. The proof of Lemma 12 is based upon an application of [14, Theorem 14] in the case $n=4$ and $\mathbf{B}=(1, B, B, B)$. Let $p$ be any prime, with $p \geq C B^{1 / \sqrt{d}+\varepsilon}$ for an appropriate constant $C$ depending at most upon $d$ and $\varepsilon$. Furthermore, let $\pi=\left[1, \pi_{1}, \pi_{2}, \pi_{3}\right] \in X_{p}\left(\mathbb{F}_{p}\right)$ be any non-singular point on the reduction $X_{p}$ of $X$ modulo $p$. Then a rudimentary examination of the proof shows that there exists a form $G_{\pi} \in \mathbb{Z}\left[X_{0}, X_{1}, X_{2}, X_{3}\right]$ of degree $O_{d, \varepsilon}(1)$ which is not divisible by $F$, such that $G_{\pi}$ vanishes at all points of the set $S_{p}(X ; B, \pi)$.

Our task is to show that there exists a set $\Pi$ of $O_{d, \varepsilon}(1)$ primes $p$ which all satisfy (6.6), such that for each $x \in S(U ; B)$ there exists $p \in \Pi$ for which the reduction $\pi$ of the point $x$ modulo $p$ lies in the open subset $U_{p}$. This will clearly suffice to establish the lemma. For $0 \leq i \leq t$ let

$$
\phi_{i}\left(X_{0}, X_{1}, X_{2}, X_{3}\right)=\Phi_{i}\left(a_{\mathbf{e}} ; X_{0}, X_{1}, X_{2}, X_{3}\right) \in \mathbb{Z}\left[X_{0}, X_{1}, X_{2}, X_{3}\right]
$$

be the forms obtained by specialising the universal polynomials in Lemma 11 at the primitive form $F$. It is clear that $t=O_{d}(1)$ and that the degree of each $\phi_{i}$ is $O_{d}(1)$. Moreover, it also follows that $\log H\left(\phi_{i}\right)=O_{d}(\log B)$ for $0 \leq i \leq t$, by (6.5). Now let $x \in S(U ; B)$. Then by the remark after Lemma 11 we may assume that there exists $0 \leq i \leq t$ such that $\phi_{i}\left(1, x_{1}, x_{2}, x_{3}\right) \neq 0$. In particular it is clear that

$$
\log \left|\phi_{i}\left(1, x_{1}, x_{2}, x_{3}\right)\right| \ll_{d} \log B
$$

Hence the number of primes $p \geq C B^{1 / \sqrt{d}+\varepsilon}$ such that $p \mid \phi_{i}\left(1, x_{1}, x_{2}, x_{3}\right)$ is at most $c(d, \varepsilon)$. By Bertrand's postulate we may select a set $\Pi$ of primes satisfying (6.6) such that $\# \Pi>c(d, \varepsilon)$. Thus $\Pi$ must contain a prime $p$ for which $p \nmid$ $\phi_{i}\left(1, x_{1}, x_{2}, x_{3}\right)$. We complete the proof of Lemma 12 by applying Lemma 11 with the choice of field $K=\mathbb{F}_{p}$.

It is clear from Lemma 12 that we shall need to consider the contribution to $N^{\text {aff }}(X ; B)$ arising from points lying on a finite set of curves that are contained 
in the surface $X$. Let $I_{D}$ be any finite set of integral curves $C$ contained in $X$, each of degree $D$, and define

$$
\Sigma_{D}=\bigcup_{C \in I_{D}} C
$$

The following result is a crucial ingredient in the proof of Theorem 2, and will be established in the following subsection. In fact it can be thought of as providing independent evidence for Conjecture 3.

Proposition 1. Let $\varepsilon>0$ and suppose that $X \subset \mathbb{P}^{3}$ is a surface of degree $d \geq 3$ such that $X \cap H_{\infty}$ is integral. Then we have

$$
N^{\text {aff }}\left(\Sigma_{D} ; B\right) \ll_{d, \varepsilon} B^{\varepsilon} \max \left\{B^{2 / d}, B^{1 / D}, \# I_{D}\right\}
$$

for $D=1$ or 2 .

\subsection{Proof of Proposition 1}

Let $X \subset \mathbb{P}^{3}$ be a surface of degree $d \geq 3$ such that $X \cap H_{\infty}$ is integral, and let $I_{D}$ be a finite set of curves $C$ contained in $X$, each of degree $D$. We begin with the case $D=1$. Any line $L \in I_{1}$ which contains at most one point $\left[1, x_{1}, x_{2}, x_{3}\right] \in \mathbb{P}^{3}(\mathbb{Z})$ such that $H\left(\left[1, x_{1}, x_{2}, x_{3}\right]\right) \leq B$, clearly contributes $O(1)$ to $N^{\text {aff }}\left(\Sigma_{1} ; B\right)$. Such lines are therefore satisfactory from the point of view Proposition 1. Suppose now that $L \in I_{1}$ contains more than one rational point $\left[1, x_{1}, x_{2}, x_{3}\right]$ of height at most $B$. We choose two such points $[1, \mathbf{t}]$ and $[1, \mathbf{t}+\mathbf{s}]$, for $\mathbf{t}, \mathbf{s} \in \mathbb{Z}^{3}$ such that $|\mathbf{s}|$ is minimal. In the notation of (6.4), it is then clear that every member of the set $S(L ; B)$ is represented by an integer vector of the form $(1, \mathbf{t}+n \mathbf{s})$, for some $n \in \mathbb{Z}$. In particular it follows from Lemma 1 that

$$
N^{\mathrm{aff}}(L ; B)=\# S(L ; B)=O(1+B /|\mathbf{s}|) .
$$

Now the vector $\mathbf{s}$ produces a rational point $[0, \mathbf{s}]$ on the integral curve $X \cap H_{\infty}$, and by Lemma 9 there are $O_{d}(1)$ lines through such a point. Thus the contribution from all the lines under consideration passing through $[0, \mathbf{s}]$ is $O_{d}(1+B /|\mathbf{s}|)$. It remains to sum over all rational points $[0, \mathbf{s}] \in X \cap H_{\infty}$ for which $|\mathbf{s}| \ll B$. We do this by considering separate dyadic ranges $S<|\mathbf{s}| \leq 2 S$. According to Lemma 4 there are at most

$$
N_{X \cap H_{\infty}}(2 S) \ll_{d, \varepsilon} S^{2 / d+\varepsilon}
$$

such points. Thus the overall contribution is $O_{d, \varepsilon}\left(S^{2 / d+\varepsilon}+B S^{2 / d-1+\varepsilon}\right)$ from this range. When we sum this up with $S \ll B$ running over powers of 2 we obtain a total $O_{d, \varepsilon}\left(B^{1+\varepsilon}\right)$, as required. This completes the argument for the case $D=1$.

Turning to the case $D=2$, we first record a basic estimate for the number of rational points of bounded height on certain conics, in which one of the coordinates is fixed. Let $C \subset \mathbb{P}^{2}$ be a plane conic defined by a non-singular 
primitive quadratic form $q \in \mathbb{Z}\left[X_{0}, X_{1}, X_{2}\right]$. Suppose that the coefficients of $q$ are bounded in modulus by $H(q)$, and that the binary form $q\left(0, X_{1}, X_{2}\right)$ is also non-singular. Then the second author has shown that

$$
\#\left\{\left[k, x_{1}, x_{2}\right] \in C \cap \mathbb{P}^{2}(\mathbb{Z}): H\left(\left[k, x_{1}, x_{2}\right]\right) \leq B\right\} \ll_{\varepsilon} H(q)^{\varepsilon} B^{\varepsilon}
$$

for any fixed integer $k$ and any choice of $\varepsilon>0[13$, Theorem 3]. We can remove the dependence of this estimate upon $H(q)$ by applying Lemma 5. Indeed we find that either there are $O(1)$ points, or else $H(q) \ll B^{12}$. In either case the total number of solutions is $O_{\varepsilon}\left(B^{13 \varepsilon}\right)$. We may therefore conclude as follows.

Lemma 13. Let $\varepsilon>0$ and suppose that $C \subset \mathbb{P}^{2}$ is a plane conic defined by a non-singular quadratic form $q \in \mathbb{Z}\left[X_{0}, X_{1}, X_{2}\right]$ such that the binary form $q\left(0, X_{1}, X_{2}\right)$ is also non-singular. Then for any integer $k$ we have

$$
\#\left\{\left[k, x_{1}, x_{2}\right] \in C \cap \mathbb{P}^{2}(\mathbb{Z}): H\left(\left[k, x_{1}, x_{2}\right]\right) \leq B\right\} \ll_{\varepsilon} B^{\varepsilon} .
$$

We are now ready to handle those conics $C \in I_{2}$ which are not tangent to the plane $H_{\infty} \in \mathbb{P}^{3^{*}}$. Such a conic is specified by a linear equation $a_{0} X_{0}=$ $a_{1} X_{1}+a_{2} X_{2}+a_{3} X_{3}$, in which not all the coefficients $a_{1}, a_{2}, a_{3}$ vanish, together with a homogeneous quadratic equation $Q\left(X_{0}, X_{1}, X_{2}, X_{3}\right)=0$. If $a_{3} \neq 0$, say, we may use the linear equation to eliminate $X_{3}$, so as to produce an equation $q\left(X_{0}, X_{1}, X_{2}\right)=0$. Since $C$ is assumed to be integral it follows that $q$ must be non-singular. Similarly, since $C$ is not tangent to $H_{\infty}$ the binary form $q\left(0, X_{1}, X_{2}\right)$ will also be non-singular. We may therefore apply Lemma 13 to deduce that

$$
\#\left\{\left[1, x_{1}, x_{2}, x_{3}\right] \in C \cap \mathbb{P}^{3}(\mathbb{Z}): H\left(\left[1, x_{1}, x_{2}, x_{3}\right]\right) \leq B\right\} \ll_{\varepsilon} B^{\varepsilon} .
$$

Thus conics which are not tangent to $H_{\infty}$ make a satisfactory contribution to $N^{\text {aff }}\left(\Sigma_{2} ; B\right)$.

It remains to consider conics $C$ which are tangent to $H_{\infty}$. We plan to show that the affine integral points on such a conic can be parameterised by the values at integer points of a small number of quadratic polynomials. To estimate how many such polynomials are necessary we must first bound the coefficients of the defining equations for $C$. As above $C$ can be given by a linear equation $a_{0} X_{0}=a_{1} X_{1}+a_{2} X_{2}+a_{3} X_{3}$, in which $a_{3}$, say, is non-zero, and a homogeneous quadratic equation in which we can eliminate $X_{3}$ to produce the defining equation $q\left(X_{0}, X_{1}, X_{2}\right)=0$ for $C$. An application of Lemma 5 shows that either $C$ contains $O(1)$ rational points of height at most $B$, or else $H(q) \ll$ $B^{12}$, as we henceforth assume. We proceed by showing that the coefficients of the linear form can be taken to satisfy

$$
\left|a_{0}\right| \ll B^{3}, \quad\left|a_{1}\right|,\left|a_{2}\right|,\left|a_{3}\right| \ll B^{2} .
$$

To do so we first consider the case in which the points $\left[1, x_{1}, x_{2}, x_{3}\right] \in \mathbb{P}^{3}(\mathbb{Z})$ of height at most $B$, which lie on the plane defined by the linear equation, are restricted to a line contained in the plane. In this case the relevant points on $C$ 
are also restricted to a line, so that there are at most two rational points to be counted. In the alternative case we can find rational points $\left[1, x_{1}^{(i)}, x_{2}^{(i)}, x_{3}^{(i)}\right]$ contained in the plane, for $1 \leq i \leq 3$, which lie in general position and have height at most $B$. It follows that one can write a suitable scalar multiple of the vector $\left(a_{0}, a_{1}, a_{2}, a_{3}\right)$ in terms of determinants in the integer vectors $\left(1, x_{1}^{(i)}, x_{2}^{(i)}, x_{3}^{(i)}\right)$. Taking h.c.f. $\left(a_{0}, a_{1}, a_{2}, a_{3}\right)=1$ this establishes (6.8).

Next we obtain a preliminary polynomial parameterisation of $C$. Since $C$ is tangent to $H_{\infty}$ the binary form $q\left(0, X_{1}, X_{2}\right)$ must have rank 1 . This allows us to write

$$
q\left(X_{0}, X_{1}, X_{2}\right)=a\left(\alpha X_{1}+\beta X_{2}\right)^{2}+b X_{0} X_{1}+c X_{0} X_{2}+d X_{0}^{2},
$$

for integers $a, b, c, d, \alpha, \beta \ll B^{12}$ with $\alpha, \beta$ coprime. Choose integers $\gamma, \delta \ll B^{12}$ with $\alpha \delta-\beta \gamma=1$ and set

$$
X_{0}=Y_{0}, \quad \alpha X_{1}+\beta X_{2}=Y_{1}, \quad \gamma X_{1}+\delta X_{2}=Y_{2},
$$

so that

$$
q\left(X_{0}, X_{1}, X_{2}\right)=q^{\prime}\left(Y_{0}, Y_{1}, Y_{2}\right)=a Y_{1}^{2}+e Y_{0} Y_{1}+f Y_{0} Y_{2}+d Y_{0}^{2}
$$

for integers $e, f \ll B^{24}$. Clearly the zero locus of $q$ coincides precisely with the zero locus of $q^{\prime}$. If $f$ were to vanish the conic $C$ would degenerate to a pair of lines, which is a case we have excluded. Hence $f \neq 0$, so that if $X_{0}=Y_{0}=1$ then

$$
Y_{2}=-f^{-1}\left(a Y_{1}^{2}+e Y_{1}+d\right)
$$

and hence

$$
X_{1}=\delta Y_{1}-\beta Y_{2}=q_{1}\left(Y_{1}\right), \quad X_{2}=\alpha Y_{2}-\gamma Y_{1}=q_{2}\left(Y_{1}\right)
$$

and

$$
X_{3}=a_{3}^{-1}\left(a_{0}-a_{1} X_{1}-a_{2} X_{2}\right)=q_{3}\left(Y_{1}\right)
$$

where $q_{1}, q_{2}, q_{3}$ are quadratic polynomials with rational coefficients whose common denominator is $O\left(B^{\kappa}\right)$ for some absolute constant $\kappa$.

Our problem is now to estimate the number of integers $Y$ for which

$$
\left(q_{1}(Y), q_{2}(Y), q_{3}(Y)\right) \in \mathbb{Z}^{3}, \quad \max \left\{\left|q_{1}(Y)\right|,\left|q_{2}(Y)\right|,\left|q_{3}(Y)\right|\right\} \leq B .
$$

If there is no such integer we are clearly done. Otherwise let $Y^{(*)}$ be any such integer and substitute $Y=Y^{(*)}+Z$ to produce polynomials $Q_{i}(Z)$ each with an integer constant term. We then write $D$ for the lowest common denominator of the coefficients of the polynomials $Q_{i}$ so that

$$
Q_{i}(Z)=A_{i}+D^{-1}\left(B_{i} Z+C_{i} Z^{2}\right), \quad A_{i}, B_{i}, C_{i} \in \mathbb{Z},
$$

for $i=1,2,3$. In view of our estimate for the common denominator of the polynomials $q_{i}$ we have

$$
D \ll B^{\kappa} .
$$


The polynomials $Q_{i}$ provide our "preliminary parameterisation".

We proceed to examine the set of integers $Z$ for which $Q_{1}(Z), Q_{2}(Z), Q_{3}(Z)$ are all integral. We classify such $Z$ according to the value of h.c.f. $(Z, D)=\lambda$, say, and we write $D=\lambda \mu$. The number of possible classes is $d(D) \ll_{\varepsilon} B^{\varepsilon}$ in view of (6.10). It is precisely for this estimate that it is necessary to control the size of the coefficients in our preliminary parameterisation. We now claim that if the class corresponding to $\lambda$ is non-empty then there exists $Z_{\lambda} \in \mathbb{Z}$ and $D_{\lambda} \mid D$ such that

$$
\begin{aligned}
\left\{Z \in \mathbb{Z}:\left(Q_{1}(Z), Q_{2}(Z), Q_{3}(Z)\right) \in \mathbb{Z}^{3} \text {, h.c.f. }(Z, D)=\lambda\right\} \\
=\left\{Z \in \mathbb{Z}: Z \equiv Z_{\lambda}\left(\bmod D_{\lambda}\right)\right\} .
\end{aligned}
$$

Once this claim is established we will write

$$
R_{i, \lambda}(t)=Q_{i}\left(Z_{\lambda}+D_{\lambda} t\right)
$$

Then the quadratic polynomials $R_{i, \lambda}(t)$ are integer valued, so that $2 R_{i, \lambda}(T) \in$ $\mathbb{Z}[T]$. Moreover any integer point on our affine conic will be of the form $\left[1, R_{1, \lambda}(t), R_{2, \lambda}(t), R_{3, \lambda}(t)\right]$ with $t \in \mathbb{Z}$ for some value of $\lambda$. This will produce the required set of integer parameterisations, one for each value of $\lambda$.

We must now verify the above claim. Write $Z=\lambda W$. Then the condition $Q_{i}(Z) \in \mathbb{Z}$ is equivalent to $\mu \mid B_{i} W+C_{i} \lambda W^{2}$. However the relation h.c.f. $(Z, D)=$ $\lambda$ implies that $\mu$ and $W$ are coprime, so that in fact $Q_{i}(Z) \in \mathbb{Z}$ if and only if $\mu \mid B_{i}+C_{i} \lambda W$. We now write $D_{i}=$ h.c.f. $\left(\mu, C_{i} \lambda\right)$ and $\mu=D_{i} \mu_{i}$. Then for each $i$ the set of solutions $W$ of the congruence $C_{i} \lambda W \equiv-B_{i}(\bmod \mu)$ is either empty (if $\left.D_{i} \nmid B_{i}\right)$, or consists of a single residue class $Z_{i, \lambda}\left(\bmod \mu_{i}\right)$. To complete the proof of the claim it suffices to observe that the intersection of the three residue classes $Z \equiv Z_{i, \lambda}\left(\bmod \mu_{i}\right)$ is either empty, or is a single residue class modulo l.c.m $\left(\mu_{1}, \mu_{2}, \mu_{3}\right)$.

We can at last complete our treatment of the case $D=2$. Consider those rational points $\left[1, x_{1}, x_{2}, x_{3}\right] \in C \cap \mathbb{P}^{3}(\mathbb{Z})$ which are given by

$$
x_{i}=A_{i}+B_{i} t+C_{i} t^{2}, \quad(i=1,2,3),
$$

for $t \in \mathbb{Z}$ and fixed $A_{i}, B_{i}, C_{i} \in \frac{1}{2} \mathbb{Z}$. Since these polynomials parameterise $C$ it follows that the point $\left[0, C_{1}, C_{2}, C_{2}\right]$ lies on $C \cap H_{\infty}$. Moreover, Lemma 1 shows that there are

$$
\ll 1+\left(\frac{B}{\max \left\{\left|C_{1}\right|,\left|C_{2}\right|,\left|C_{3}\right|\right\}}\right)^{1 / 2}
$$

corresponding points belonging to the set $S(C ; B)$, in the notation of (6.4). Now let $p=[0, a, b, c] \in X \cap H_{\infty}$, with h.c.f. $(a, b, c)=1$, and suppose that $S<\max \{|a|,|b|,|c|\} \leq 2 S$. Then if $C \subset X$ is tangent to $H_{\infty}$ at $p=[0, a, b, c]$ it follows that the contribution from $C$ to $N^{\text {aff }}\left(\Sigma_{2} ; B\right)$ is $O_{\varepsilon}\left(B^{\varepsilon}\left(1+B^{1 / 2} S^{-1 / 2}\right)\right)$, after allowing for the various parameterisations of $C$. According to Lemma 9 we get the overall bound $O_{d, \varepsilon}\left(B^{\varepsilon}\left(1+B^{1 / 2} S^{-1 / 2}\right)\right)$ when we include all other conics in $X$ which are tangent to $H_{\infty}$ at $p$. It remains to sum over all rational points 
$p$ on $X \cap H_{\infty}$ for which $\max \{|a|,|b|,|c|\} \ll B$. We do this by the same dyadic range decomposition as was used for the case $D=1$. Each range of length $S$ will contribute

$$
\ll_{d, \varepsilon} B^{\varepsilon}\left(S^{2 / d+\varepsilon}+B^{1 / 2} S^{2 / d-1 / 2+\varepsilon}\right),
$$

giving a total contribution $O_{d, \varepsilon}\left(B^{2 \varepsilon}\left(B^{2 / d}+B^{1 / 2}\right)\right)$ to $N^{\text {aff }}\left(\Sigma_{2} ; B\right)$. This is plainly satisfactory for Proposition 1.

\subsection{Completion of the proof of Theorem 2}

Let $F \in \mathbb{Z}\left[X_{0}, X_{1}, X_{2}, X_{3}\right]$ be a primitive form of degree $d \geq 3$, defining an integral surface $X \subset \mathbb{P}^{3}$. Let $H_{\infty} \in \mathbb{P}^{3 *}$ denote the plane $X_{0}=0$. In this section we shall establish the estimate (6.3), and so complete the proof of Theorem 2. We therefore assume henceforth that the intersection $X \cap H_{\infty}$ is integral. Suppose first that the coefficients of $F$ are large compared with $B$. Then an application of Lemma 5 reveals that $N^{\text {aff }}(X ; B) \leq N^{\text {aff }}(Y ; B)$, for some curve $Y \subset \mathbb{P}^{3}$ obtained by intersecting $X$ with a distinct surface $F^{\prime}=0$. The contribution from irreducible components of $Y$ of degree 2 or more is $O_{d, \varepsilon}\left(B^{1+\varepsilon}\right)$, by Lemma 4, while for components of degree 1 the bound (6.7) again gives a satisfactory result. We henceforth assume that (6.5) holds for $F$.

Recall the definition of the non-empty open subset $U \subset X$, introduced at the close of $\S 5$. Then by the comments made directly after Lemma 11 we may argue as above to deduce that $N^{\text {aff }}(X \backslash U ; B) \ll_{d, \varepsilon} B^{1+\varepsilon}$. Hence it is sufficient to establish the estimate

$$
N^{\mathrm{aff}}(U ; B) \ll_{d, \varepsilon} \begin{cases}B^{5 /(3 \sqrt{3})+1 / 4+\varepsilon}, & d=3 \\ B^{1+\varepsilon}+B^{3 /(2 \sqrt{d})+1 / 3+\varepsilon}, & d \geq 4\end{cases}
$$

We are now in a position to apply Lemma 12 . Thus there exists a set $\Pi$ of $O_{d, \varepsilon}(1)$ primes $p$, with (6.6) holding, such that

$$
N^{\mathrm{aff}}(U ; B) \leq \sum_{p \in \Pi} \sum_{\pi \in U_{p}\left(\mathbb{F}_{p}\right)} \#\left\{\left[1, x_{1}, x_{2}, x_{3}\right] \in S_{p}(U ; B, \pi): G_{\pi}\left(1, x_{1}, x_{2}, x_{3}\right)=0\right\} .
$$

Here the $G_{\pi} \in \mathbb{Z}\left[X_{0}, X_{1}, X_{2}, X_{3}\right]$ are a finite set of forms indexed by points $\pi=\left[1, \pi_{1}, \pi_{2}, \pi_{3}\right] \in U_{p}\left(\mathbb{F}_{p}\right)$. For each $\pi \in U_{p}\left(\mathbb{F}_{p}\right)$, the form $G_{\pi}$ has degree $O_{d, \varepsilon}(1)$ and is not divisible by $F$.

Let $Y=Y_{\pi} \subset \mathbb{P}^{3}$ be an integral component of the curve $F=G_{\pi}=0$, and let

$$
N_{p}^{\mathrm{aff}}(Y ; B, \pi)=\# S_{p}(Y ; B, \pi)
$$

If $Y_{1} \cup \cdots \cup Y_{s}$ denotes the irreducible decomposition of $F=G_{\pi}=0$, with its reduced scheme structure, then we clearly have $s=O_{d, \varepsilon}(1)$ and

$$
\#\left\{\left[1, x_{1}, x_{2}, x_{3}\right] \in S_{p}(U ; B, \pi): G_{\pi}\left(1, x_{1}, x_{2}, x_{3}\right)=0\right\} \leq \sum_{1 \leq j \leq s} N_{p}^{\mathrm{aff}}\left(Y_{j} ; B, \pi\right) .
$$


As we vary over primes $p \in \Pi$ and points $\pi \in U_{p}\left(\mathbb{F}_{p}\right)$, let $I$ denote the set of integral components of the curves $F=G_{\pi}=0$ which have degree at most 2 . Since $\# U_{p}\left(\mathbb{F}_{p}\right) \leq \# X_{p}\left(\mathbb{F}_{p}\right)=O_{d}\left(p^{2}\right)$, it is clear from (6.6) that

$$
\# I \leq \# \Pi \cdot \max _{p} \# U_{p}\left(\mathbb{F}_{p}\right)=O_{d, \varepsilon}\left(B^{2 / \sqrt{d}+2 \varepsilon}\right) .
$$

Hence Proposition 1 implies that the overall contribution to $N^{\text {aff }}(U ; B)$ from the set $I$ is

$$
\ll_{d, \varepsilon} B^{1+\varepsilon}+B^{2 / \sqrt{d}+3 \varepsilon} .
$$

This is satisfactory for (6.11) provided that $d \geq 3$.

We henceforth fix a choice of prime $p \in \Pi$, and a point $\pi \in U_{p}\left(\mathbb{F}_{p}\right)$. Let $G_{\pi}$ be the corresponding form produced by Lemma 12, and let $Y=Y_{\pi} \subset \mathbb{P}^{3}$ be any integral component of the curve $F=G_{\pi}=0$, of degree $e$. It henceforth suffices to assume that $Y$ has dimension 1 and degree $e \geq 3$, since we have already taken care of the remaining components. In particular we recall that $e=O_{d, \varepsilon}(1)$. The main work in this section will be taken up with establishing the following estimate.

Proposition 2. Assume that $Y$ has degree $e \geq 3$. Then we have

$$
N_{p}^{\text {aff }}(Y ; B, \pi) \ll_{d, \varepsilon} B^{1 / e-1 /((e-1) \sqrt{d})} .
$$

When $d \geq 4$ the estimate in Proposition 2 takes its largest value at $e=3$, whereas when $d=3$ it is maximal at $e=4$. We therefore have

$$
N_{p}^{\text {aff }}(Y ; B, \pi) \ll_{d, \varepsilon} \begin{cases}B^{1 / 4-1 /(3 \sqrt{3})}, & d=3, \\ B^{1 / 3-1 /(2 \sqrt{d})}, & d \geq 4,\end{cases}
$$

whenever $Y$ has degree $e \geq 3$. On inserting this bound into our previous argument and re-defining $\varepsilon$, we therefore arrive at the inequality (6.11). Hence it will suffice to prove Proposition 2 in order to complete the proof of Theorem 2 .

We shall prove Proposition 2 by using a generalisation of the determinant method, as developed by the second author [14, Theorem 14]. By a result of Gruson, Lazarsfeld and Peskine [10], the ideal of $Y$ is generated by forms $F_{1}, \ldots, F_{r}$ whose maximal degree is bounded in terms of $e$ alone. We may also assume that these forms are linearly independent, and that the number of forms is bounded in terms of $e=O_{d, \varepsilon}(1)$. By applying Broberg's generalisation [2, Lemma 5] of Lemma 5, we may assume that either $\log H\left(F_{i}\right)=O_{d, \varepsilon}(\log B)$ for $1 \leq i \leq r$, or else

$$
N_{p}^{\text {aff }}(Y ; B, \pi)=O_{d, \varepsilon}(1)
$$

which is satisfactory for the statement of Proposition 2. We henceforth assume that the forms defining $Y$ all have logarithmic height $O_{d, \varepsilon}(\log B)$.

Let $q$ be a prime, and let $J=\left(F_{1}, \ldots, F_{r}\right) \subset \mathbb{Q}\left[X_{0}, X_{1}, X_{2}, X_{3}\right]$ be the homogeneous prime ideal defining $Y$. We write $Y_{q}=\operatorname{Proj}\left(\mathbb{F}_{q}\left[X_{0}, X_{1}, X_{2}, X_{3}\right] / J_{q}\right)$, where $J_{q}$ denotes the image of $J \cap \mathbb{Z}_{(q)}\left[X_{0}, X_{1}, X_{2}, X_{3}\right]$ in $\mathbb{F}_{q}\left[X_{0}, X_{1}, X_{2}, X_{3}\right]$. Here, as throughout this paper, $\mathbb{Z}_{(q)}$ denotes the localisation of $\mathbb{Z}$ at the prime 
$q$. Let $V \subset Y$ be the non-empty open subset of non-singular points on the curve $Y$. We shall write $V_{q}$ for the set of non-singular points on $Y_{q}$. Finally, for any choice of $\omega=\left[1, \omega_{1}, \omega_{2}, \omega_{3}\right] \in V_{q}\left(\mathbb{F}_{q}\right)$ we set

$$
S_{p, q}(V ; B, \pi, \omega)=\left\{x \in S_{p}(V ; B, \pi): x_{i} \equiv \omega_{i}(\bmod q),(1 \leq i \leq 3)\right\} .
$$

We recall here that $x=\left[1, x_{1}, x_{2}, x_{3}\right]$ for $x_{i} \in \mathbb{Z}$ such that $x_{i} \equiv \pi_{i}(\bmod p)$ for $1 \leq i \leq 3$, whenever $x \in S_{p}(V ; B, \pi)$. We proceed by establishing the following result.

Lemma 14. Let $a>0$. Then there exists a set $\Omega$ of $O_{d, \varepsilon}(1)$ primes $q$, with $p \notin \Omega$ and

$$
B^{a} \ll_{d, \varepsilon} q \ll_{d, \varepsilon} B^{a},
$$

such that

$$
S_{p}(V ; B, \pi)=\bigcup_{q \in \Omega} \bigcup_{\substack{\omega \in V_{q}\left(\mathbb{F}_{q}\right) \\ \omega=\left[1, \omega_{1}, \omega_{2}, \omega_{3}\right]}} S_{p, q}(V ; B, \pi, \omega) .
$$

Proof. The proof of Lemma 14 is straightforward. Recall that the ideal $J$ of $Y$ is generated by $O_{d, \varepsilon}(1)$ forms of maximal degree $O_{d}(1)$, all of which have logarithmic height $O_{d, \varepsilon}(\log B)$. Using the Jacobian criterion for a point of $Y$ to be contained in the singular locus, we may find $O_{d, \varepsilon}(1)$ non-zero forms

$$
\Psi_{1}, \ldots, \Psi_{l} \in \mathbb{Z}\left[X_{0}, X_{1}, X_{2}, X_{3}\right]
$$

of maximal degree $O_{d, \varepsilon}(1)$, such that $\Psi_{i}\left(1, x_{1}, x_{2}, x_{3}\right) \neq 0$ for some $1 \leq i \leq l$ whenever $x=\left[1, x_{1}, x_{2}, x_{3}\right] \in S_{p}(V ; B, \pi)$. On noting that

$$
\log \left|\Psi_{i}\left(1, x_{1}, x_{2}, x_{3}\right)\right| \ll_{d, \varepsilon} \log B
$$

and recalling from (6.6) that $\log p \ll_{d, \varepsilon} \log B$, one easily deduces the statement of Lemma 14 along precisely the same lines as those used to prove Lemma 12 .

It follows from the proof of Lemma 14 that $Y \backslash V$ is a variety of dimension 0 and degree $O_{d, \varepsilon}(1)$, whence $N_{p}^{\text {aff }}(Y \backslash V ; B, \pi)=O_{d, \varepsilon}(1)$. In order to prove Proposition 2 it therefore suffices to establish the estimate

$$
N_{p}^{\mathrm{aff}}(V ; B, \pi) \ll_{d, \varepsilon} B^{1 / e-1 /((e-1) \sqrt{d})} .
$$

To do so we shall apply Lemma 14 with $a=1 / e-1 /((e-1) \sqrt{d})$, so that in particular

$$
B^{1 / e-1 /((e-1) \sqrt{d})} \ll_{d, \varepsilon} q \ll_{d, \varepsilon} B^{1 / e-1 /((e-1) \sqrt{d})},
$$

for every $q \in \Omega$. On noting that $\# V_{q}\left(\mathbb{F}_{q}\right) \leq \# Y_{q}\left(\mathbb{F}_{q}\right)=O_{d}(q)$, it plainly suffices to show that

$$
S_{p, q}(V ; B, \pi, \omega) \ll_{d, \varepsilon} 1,
$$

for each $q \in \Omega$ and each corresponding point $\omega \in V_{q}\left(\mathbb{F}_{q}\right)$. 
We henceforth fix a choice of $q \in \Omega$, and a point $\omega=\left[1, \omega_{1}, \omega_{2}, \omega_{3}\right] \in V_{q}\left(\mathbb{F}_{q}\right)$. We shall establish the existence of an auxiliary form, not contained in the prime ideal $J$ defining $Y$, which vanishes at all points $S_{p, q}(V ; B, \pi, \omega)$. An application of Bézout's theorem will then yield the estimate (6.13), provided we can show that the degree of this auxiliary form is bounded in terms of $d$ and $\varepsilon$ alone.

For a fixed positive integer $k$, we shall need to find a set of monomials $M_{1}, \ldots, M_{k} \in \mathbb{Z}\left[X_{0}, X_{1}, X_{2}, X_{3}\right]$ of degree $D$ depending only on $e$ and $k$, such that no non-trivial linear combination of them belongs to the prime ideal $J$, and such that the sum

$$
\operatorname{deg} M_{1}\left(1, T_{1}, T_{2}, T_{3}\right)+\cdots+\operatorname{deg} M_{k}\left(1, T_{1}, T_{2}, T_{3}\right)
$$

is as small as possible. We shall henceforth write $m_{i}\left(T_{1}, T_{2}, T_{3}\right)$ to denote the monomial $M_{i}\left(1, T_{1}, T_{2}, T_{3}\right)$, for $1 \leq i \leq k$. The following result shows that such a set always exists provided that $k$ is taken to be sufficiently large.

Lemma 15. Let $k \gg_{e} 1$ be a positive integer. Then there exists a set $\mathcal{M}(J, k)$ of monomials $M_{1}, \ldots, M_{k} \in \mathbb{Z}\left[X_{0}, X_{1}, X_{2}, X_{3}\right]$ of degree $D=O_{d, k}(1)$, such that no non-trivial linear combination of them belongs to the prime ideal $J$, and such that

$$
\operatorname{deg} m_{1}+\cdots+\operatorname{deg} m_{k} \leq \frac{k^{2}}{2 e}+O_{e}(k) .
$$

Proof. Let $H_{\infty} \in \mathbb{P}^{3^{*}}$ be the plane $X_{0}=0$, and let $Z$ be the scheme-theoretic intersection of $Y$ and $H_{\infty}$. Then there exists an integer $\delta_{0}$ depending only on $e$, such that the Hilbert function $h_{Z}(\delta)$ of $Z \subset \mathbb{P}^{3}$ satisfies $h_{Z}(\delta)=e$ for $\delta \geq \delta_{0}$. Whenever $\delta \geq \delta_{0}$ we may therefore find $e$ monomials in $\mathbb{Z}\left[X_{1}, X_{2}, X_{3}\right]$ of degree $\delta$, such that no non-trivial linear combination of them belongs to the ideal in $\mathbb{Q}\left[X_{0}, X_{1}, X_{2}, X_{3}\right]$ which is generated by $J$ and $X_{0}$. Denote these monomials by $m_{1, \delta}, m_{2, \delta}, \ldots, m_{e, \delta}$. We then examine the monomials

$$
M_{i, \delta}=X_{0}^{D-\delta} m_{i, \delta}\left(X_{1}, X_{2}, X_{3}\right), \quad\left(1 \leq i \leq e, \quad \delta_{0} \leq \delta \leq D\right) .
$$

Suppose that some linear combination of them belonged to $J$. We would then have a relation of the form

$$
\sum_{\delta_{0} \leq \delta \leq D} X_{0}^{D-\delta} R_{\delta}\left(X_{1}, X_{2}, X_{3}\right) \in J
$$

where $R_{\delta}$ is a linear combination of the monomials $m_{1, \delta}, m_{2, \delta}, \ldots, m_{e, \delta}$. By our construction, if $R_{\delta} \in\left\langle J, X_{0}\right\rangle$ then the coefficients of $m_{1, \delta}, \ldots, m_{e, \delta}$ must all vanish, so that $R_{\delta}=0$. Using this observation for $R_{D}, R_{D-1}, R_{D-2}, \ldots$ we find that no non-trivial linear combination of the $M_{i, \delta}$ can belong to $J$.

Writing $n_{D}$ for the number of monomials under consideration we have $n_{D}=$ $e D+O_{e}(1)$. Moreover the sum of the degrees of the corresponding $m_{i, \delta}$ is $e D^{2} / 2+O_{e}(D)$. The result then follows on choosing $D$ to be the smallest integer for which $n_{D} \geq k$, and ignoring any surplus monomials. 
Let $k \gg_{e} 1$ be a positive integer and suppose that $\# S_{p, q}(V ; B, \pi, \omega) \geq k$. We select a list of points

$$
x^{(1)}, \ldots, x^{(k)} \in S_{p, q}(V ; B, \pi, \omega) .
$$

Then, for each $1 \leq j \leq k$, we have $x^{(j)}=\left[1, x_{1}^{(j)}, x_{2}^{(j)}, x_{3}^{(j)}\right]$ for $x_{i}^{(j)} \in \mathbb{Z}$ such that

$$
x_{i}^{(j)} \equiv \pi_{i} \quad(\bmod p), \quad x_{i}^{(j)} \equiv \omega_{i} \quad(\bmod q), \quad(i=1,2,3) .
$$

We define $\Delta$ to be the determinant of the $k \times k$ matrix

$$
\left(M_{i}\left(1, x_{1}^{(j)}, x_{2}^{(j)}, x_{3}^{(j)}\right)\right)_{1 \leq i, j \leq k},
$$

where $M_{1}, \ldots, M_{k} \in \mathcal{M}(J, k)$, so that in particular we have

$$
\Delta=\operatorname{det}\left(m_{i}\left(x_{1}^{(j)}, x_{2}^{(j)}, x_{3}^{(j)}\right)\right)_{1 \leq i, j \leq k} .
$$

Thus it follows from an application of Lemma 15 that

$$
\log |\Delta| \leq k \log k+\frac{k^{2}}{2 e} \log B+O_{e}(k \log B) .
$$

We shall also need to show that the determinant $\Delta$ is divisible by large powers of $p$ and $q$. Once combined with (6.16), this information will then be used to establish that $\Delta=0$ provided that $k$ is chosen to be sufficiently large in terms of $d$ and $\varepsilon$. In particular, once such a value of $k$ is fixed, it will follow that the monomials in $\mathcal{M}(J, k)$ will all have degree $O_{d, \varepsilon}(1)$. Before carrying out this plan, we first show how (6.13) will follow. Set $\# S_{p, q}(V ; B, \pi, \omega)=N$, and consider the matrix

$$
\mathbf{M}=\left(m_{i}\left(x_{1}^{(j)}, x_{2}^{(j)}, x_{3}^{(j)}\right)\right)_{1 \leq i \leq k, 1 \leq j \leq N} .
$$

Then either $N<k$, or we may assume that $N \geq k$ and so $\Delta=0$ by assumption. In either case it follows therefore that $\mathbf{M}$ has rank at most $k-1$. Hence we may find a non-trivial linear combination $M$ of the monomials $M_{1}, \ldots, M_{k}$ which vanishes at every point of $S_{p, q}(V ; B, \pi, \omega)$, but not at all points of $Y$. But then an application of Bézout's theorem yields

$$
\# S_{p, q}(V ; B, \pi, \omega) \leq e \operatorname{deg}(M)
$$

which thereby establishes (6.13).

It remains to establish that $\Delta=0$ provided that $k$ is chosen to be sufficiently large in terms of $d$ and $\varepsilon$. For this we use the fact that (6.15) holds for the points $x_{1}, \ldots, x_{k}$ that we are interested in, where $\pi=\left[1, \pi_{1}, \pi_{2}, \pi_{3}\right] \in \mathbb{P}^{3}\left(\mathbb{F}_{p}\right)$ is a nonsingular point on $X_{p}$ which is of multiplicity at most 2 on the intersection with the tangent plane to $X_{p}$ at $\pi$, and $\omega=\left[1, \omega_{1}, \omega_{2}, \omega_{3}\right] \in \mathbb{P}^{3}\left(\mathbb{F}_{q}\right)$ is a non-singular point on the curve $Y_{q}$. The main obstacle that we shall have to overcome is the fact that $\pi$ need not be a non-singular point on the curve $Y_{p}$, despite being non-singular on $X_{p}$. It is in the following result that we make crucial use of the fact that $\pi$ is of multiplicity at most 2 on the tangent plane section. 
Lemma 16. For any $k \in \mathbb{N}$ we have $p^{\alpha(k)} q^{\beta(k)} \mid \Delta$, where

$$
\alpha(k) \geq \frac{k^{2}}{2(e-1)}-O_{d, e, \varepsilon}(k), \quad \beta(k)=\frac{k(k-1)}{2} .
$$

Proof. Let $T_{i}=X_{i} / X_{0}$ for $1 \leq i \leq 3$, and let $I \subset \mathbb{Q}\left[T_{1}, T_{2}, T_{3}\right]$ be the ideal generated by the polynomials whose homogenisation belongs to the ideal $J$ of $Y$. We begin by showing that $p^{\alpha(k)} \mid \Delta$. Let $I_{p}$ be the image of $I \cap \mathbb{Z}_{(p)}\left[T_{1}, T_{2}, T_{3}\right]$ in $\mathbb{F}_{p}\left[T_{1}, T_{2}, T_{3}\right]$, where $\mathbb{Z}_{(p)}$ denotes the localisation of $\mathbb{Z}$ at the prime $p$. Let $A=A_{p}$ be the stalk of the curve $Y_{p}$ at the point $\pi=\left[1, \pi_{1}, \pi_{2}, \pi_{3}\right] \in Y_{p}\left(\mathbb{F}_{p}\right)$, and let $\mathfrak{m}$ be its maximal ideal. After a linear change of variables we may in fact assume that $\pi=[1,0,0,0]$ and that the tangent plane of $X_{p}$ at $\pi$ is given by $X_{3}=0$.

We now apply a rather general result due to the third author, which can be found in the appendix. Let $\left(n_{\ell}(A)\right)_{\ell=1}^{\infty}$ be the sequence of integers $n \geq 0$, in which each $n$ occurs precisely $\operatorname{dim}_{A / \mathfrak{m}} \mathfrak{m}^{n} / \mathfrak{m}^{n+1}$ times. Then it follows that $p^{\mathcal{A}(k)}$ divides $\Delta$, where

$$
\mathcal{A}(k)=n_{1}(A)+\cdots+n_{k}(A) .
$$

We need to show that

$$
\mathcal{A}(k) \geq \frac{k^{2}}{2(e-1)}-O_{d, e, \varepsilon}(k),
$$

for any $k \in \mathbb{N}$. Let $B=B_{p}$ be the stalk of $X_{p}$ at $\pi$, and let $\mathfrak{n}$ be the maximal ideal of this local ring. Then $B$ is a regular local ring of Krull dimension 2 since $\pi$ is a non-singular point on $X_{p}$. There is also a surjective ring homomorphism $\kappa: B \rightarrow A$ induced by the embedding of $Y_{p}$ in $X_{p}$. Hence it follows that

$$
\operatorname{dim}_{A / \mathfrak{m}} \mathfrak{m}^{n} / \mathfrak{m}^{n+1} \leq \operatorname{dim}_{B / \mathfrak{n}} \mathfrak{n}^{n} / \mathfrak{n}^{n+1}=n+1
$$

for any $n \in \mathbb{N}$. We now show that there exists an integer $n_{0}$ depending only on $d$ and $\varepsilon$, such that

$$
\operatorname{dim}_{A / \mathfrak{m}} \mathfrak{m}^{n} / \mathfrak{m}^{n+1} \leq e-1,
$$

for all $n \geq n_{0}$. It is clear that this will suffice to establish (6.18), via (6.17).

Recall that the prime ideal $J \subset \mathbb{Q}\left[X_{0}, X_{1}, X_{2}, X_{3}\right]$ of $Y$ is generated by $O_{d, \varepsilon}(1)$ forms of maximal degree $O_{d, \varepsilon}(1)$. We proceed to show that the same is true of the ideal $J_{p} \subset \mathbb{F}_{p}\left[X_{0}, X_{1}, X_{2}, X_{3}\right]$. Now there are only $O_{d, \varepsilon}(1)$ possible Hilbert functions for $J$ by [2, Lemma 1], and it is not hard to see that $J \subset$ $\mathbb{Q}\left[X_{0}, X_{1}, X_{2}, X_{3}\right]$ and $J_{p} \subset \mathbb{F}_{p}\left[X_{0}, X_{1}, X_{2}, X_{3}\right]$ have the same Hilbert function since $\left(J \cap \mathbb{Z}_{(p)}\left[X_{0}, X_{1}, X_{2}, X_{3}\right]\right)_{D}$ is a pure sublattice of $\left(\mathbb{Z}_{(p)}\left[X_{0}, X_{1}, X_{2}, X_{3}\right]\right)_{D}$ for each grade $D$. It follows that there are only $O_{d, \varepsilon}(1)$ possible Hilbert functions for $J_{p}$, and so only $O_{d, \varepsilon}(1)$ possible Hilbert polynomials. In order to deduce that $J_{p}$ is generated by forms of maximal degree $O_{d, \varepsilon}(1)$, it is therefore enough to prove that $J_{p}$ is generated by forms of degree bounded solely in terms of the Hilbert polynomial for $J_{p}$. But this is a standard fact from the theory of Hilbert schemes (see [15, Theorem I.1.5], for example). 
We now consider the tangent cone $\mathrm{TC}_{\pi}\left(Y_{p}\right)$ of $Y_{p}$ at $\pi$. Let $G_{1}, \ldots, G_{s} \in$ $\mathbb{F}_{p}\left[X_{0}, X_{1}, X_{2}, X_{3}\right]$ be forms of degree $O_{d, \varepsilon}(1)$, which together with $F$ define $Y_{p} \subset \mathbb{P}_{\mathbb{F}_{p}}^{3}$, and let $g_{i}\left(T_{1}, T_{2}\right)=G_{i}\left(1, T_{1}, T_{2}, 0\right)$ for $1 \leq i \leq s$. We may then write each $g_{i}$ as a finite sum of forms in the variables $T_{1}, T_{2}$. Let $h_{i}\left(T_{1}, T_{2}\right)$ be the term of lowest degree in such an expansion of $g_{i}$. If $g_{i}=0$ we take $h_{i}=0$. The tangent cone $\mathrm{TC}_{\pi}\left(Y_{p}\right)$ then has a concrete description as the affine subscheme of $\mathrm{TC}_{\pi}\left(X_{p}\right)=\operatorname{Spec}\left[T_{1}, T_{2}\right]$ cut out by the forms $h_{1}\left(T_{1}, T_{2}\right), \ldots, h_{s}\left(T_{1}, T_{2}\right)$. This is described in $[16, \S I I I .3]$, for example. The coordinate ring $\mathbb{F}_{p}\left[T_{1}, T_{2}\right] /\left(h_{1}, \ldots, h_{s}\right)$ has a natural grading and it follows from [2, Lemma 1] that the Hilbert function $\phi(n)$ of the corresponding homogeneous coordinate ring is equal to a polynomial $P(n)$ for all $n \geq n_{0}$, where $n_{0}$ is an integer bounded in terms of the maximum degree of $h_{1}, \ldots, h_{s}$. We therefore have $n_{0}=O_{d, \varepsilon}(1)$.

In fact there is a more intrinsic description [16, §III.3] of the homogeneous coordinate ring $\mathbb{F}_{p}\left[T_{1}, T_{2}\right] /\left(h_{1}, \ldots, h_{s}\right)$. It is the graded ring

$$
\operatorname{gr}(A)=\bigoplus_{n=0}^{\infty} \mathfrak{m}^{n} / \mathfrak{m}^{n+1},
$$

with $\mathfrak{m}^{0}=A$. In particular, $\phi(n)=\operatorname{dim}_{A / \mathfrak{m}} \mathfrak{m}^{n} / \mathfrak{m}^{n+1}$ for all $n \geq 0$. We may now complete the proof of (6.19). Since $Y_{p}$ has dimension 1 it follows that the multiplicity $\operatorname{mult}_{\pi}\left(Y_{p}\right)$ of $Y_{p}$ at $\pi$ is equal to the dimensions $\phi(n)$ of the vector spaces $\mathfrak{m}^{n} / \mathfrak{m}^{n+1}$ over $A / \mathfrak{m}$ for all sufficiently large $n$ (see Fulton [7, Excercise 4.3.1], for example). It therefore follows that $P(n)$ must be a constant polynomial and $\operatorname{dim}_{A / \mathfrak{m}} \mathfrak{m}^{n} / \mathfrak{m}^{n+1}=\operatorname{mult}_{\pi}\left(Y_{p}\right)$ for all $n \geq n_{0}$. We clearly have the trivial upper bound

$$
\operatorname{mult}_{\pi}\left(Y_{p}\right) \leq \operatorname{deg} Y_{p}=\operatorname{deg} Y=e .
$$

Suppose now that $\operatorname{mult}_{\pi}\left(Y_{p}\right)=e$. Then $Y_{p}$ must be a cone with vertex $\pi$. In particular $Y_{p}$ is contained in the intersection of $X_{p}$ with its tangent plane at $\pi$. This contradicts the assumption that $\pi$ has multiplicity at most $2<e$ on the tangent plane section of $X_{p}$ at $\pi$, and so establishes (6.19).

It remains to show that $q^{\beta(k)} \mid \Delta$. Indeed, since $p \notin \Omega$ by Lemma 14 it will then follow that $p^{\alpha(k)} q^{\beta(k)} \mid \Delta$, as required. Adopting the same notation as above, we let $A=A_{q}$ be the stalk of the curve $Y_{q}$ at the non-singular point $\omega=\left[1, \omega_{1}, \omega_{2}, \omega_{3}\right] \in Y_{q}\left(\mathbb{F}_{q}\right)$, and let $\mathfrak{m}$ be its maximal ideal. Then an application of the result in the appendix yields

$$
q^{n_{1}(A)+\cdots+n_{k}(A)} \mid \Delta .
$$

It is then a simple matter to note that $n_{1}(A)+\cdots+n_{k}(A)=\beta(k)$ in this case. Indeed for any $n \geq 0$ the dimension of $\mathfrak{m}^{n} / \mathfrak{m}^{n+1}$ over $A / \mathfrak{m}$ is now equal to the multiplicity of the non-singular point $\omega$ on the reduced curve $Y_{q}$, which is plainly equal to 1 .

We now have all of the tools with which to conclude the proof of Theorem 2. Recall the inequality (6.6) for $p$. On combining Lemma 16 with the upper bound 
(6.16) for $\log |\Delta|$, we easily deduce that $\Delta=0$ whenever

$$
\log q>\left(\frac{1}{e}-\frac{1}{(e-1) \sqrt{d}}\right) \log B-\frac{\varepsilon \log B}{e-1}+O_{d, e, \varepsilon}(1)+O_{d, e, \varepsilon}\left(\frac{\log B}{k-1}\right) .
$$

Upon taking $k$ to be sufficiently large compared with $d$ and $\varepsilon$, this is plainly satisfied for every $q \in \Omega$, by (6.12).

\section{A Appendix}

Let $p$ be a prime and let $\mathbb{Z}_{(p)}$ denote the localisation of $\mathbb{Z}$ at $p$. Let $R$ be any commutative noetherian local ring containing $\mathbb{Z}_{(p)}$ as a subring. We write $A$ for the quotient ring $R / p R$, and let $\mathfrak{m}$ be the maximal ideal of $A$. Finally, let

$$
n_{1}(A), n_{2}(A), n_{3}(A), \ldots
$$

be the non-decreasing sequence of integers $n \geq 0$ in which each $n$ occurs precisely $\operatorname{dim}_{A / \mathfrak{m}} \mathfrak{m}^{n} / \mathfrak{m}^{n+1}$ times. The purpose of this appendix is to record the following result due to the third author [18].

Lemma. Let $\psi_{1}, \ldots, \psi_{k}$ be ring homomorphisms from $R$ to $\mathbb{Z}_{(p)}$, for some positive integer $k$, and let $r_{1}, \ldots, r_{k} \in R$. Then it follows that

$$
p^{n_{1}(A)+\cdots+n_{k}(A)} \mid \operatorname{det}\left(\psi_{i}\left(r_{j}\right)\right)_{1 \leq i, j \leq k} .
$$

The main idea in the proof of this result is to replace each $r_{j}$ by a suitable linear combination $\lambda_{1} r_{1}+\cdots+\lambda_{j-1} r_{j-1}+r_{j}$, with coefficients in $\mathbb{Z}_{(p)}$, such that $\psi_{1}\left(r_{j}\right), \ldots, \psi_{k}\left(r_{j}\right)$ are all divisible by $p^{n_{j}(A)}$.

\section{References}

[1] E. Bombieri and J. Pila, The number of integral points on arcs and ovals, Duke Math. J., 59 (1989), 337-357.

[2] N. Broberg, A note on a paper by Heath-Brown: "The density of rational points on curves and surfaces", J. reine angew. Math., 571 (2004), 159-178.

[3] N. Broberg and P. Salberger, Counting rational points on threefolds, 105120. Arithmetic of higher-dimensional algebraic varieties, Progress in Mathematics 226, Birkhäuser, 2003.

[4] T.D. Browning and D.R. Heath-Brown, Counting rational points on hypersurfaces, J. reine angew. Math., to appear.

[5] D. Eisenbud and J. Harris, The Geometry of Schemes, Springer-Verlag, 2000.

[6] G. Fischer, Plane Algebraic Curves, American Mathematical Society, 2001. 
[7] W. Fulton, Intersection Theory, 2nd ed., Springer-Verlag, 1998.

[8] A. Grothendieck, Éléments de géométrie algébrique. IV. Étude locale des schémas et des morphismes de schémas IV, Inst. Hautes Études Sci. Publ. Math., 32 (1967), 361 pp.

[9] A. Grothendieck and J. Dieudonné, Éléments de géométrie algébrique. I. Grundlehren der Math. Wissenschaften 166, Springer-Verlag (1971).

[10] L. Gruson, R. Lazarsfeld and C. Peskine, On a theorem of Castelnuovo, and the equations defining space curves, Invent. Math., 72 (1983), no. 3, 491-506.

[11] J. Harris, Algebraic Geometry, Springer-Verlag, 1992.

[12] R. Hartshorne, Algebraic Geometry, Springer-Verlag, 1977.

[13] D.R. Heath-Brown, The density of rational points on cubic surfaces, Acta Arith., 79 (1997), 17-30.

[14] D.R. Heath-Brown, The density of rational points on curves and surfaces, Annals of Math., 155 (2002), 553-595.

[15] J. Kollár, Rational curves on algebraic varieties, Springer-Verlag, 1996.

[16] D. Mumford, The red book of varieties and schemes, Lect. Notes in Math. 1358, 2nd ed., Springer-Verlag, 1999.

[17] J. Pila, Density of integral and rational points on varieties, Astérisque, 228 (1995), 183-187.

[18] P. Salberger, On the density of rational and integral points on algebraic varieties, in preparation. 